\title{
Additional Findings from the Common Research Model Natural Laminar Flow Wind Tunnel Test
}

\author{
Michelle N. Lynde ${ }^{1}$, Richard L. Campbell ${ }^{2}$, and Sally A. Viken ${ }^{3}$ \\ NASA Langley Research Center, Hampton, Virginia, 23681
}

\begin{abstract}
An experimental investigation of the Common Research Model with Natural Laminar Flow (CRM-NLF) took place in the National Transonic Facility (NTF) at the NASA Langley Research Center in 2018. The 5.2\% scale semispan model was designed using a new natural laminar flow design method, Crossflow Attenuated NLF (CATNLF). CATNLF enables laminar flow on typical transport wings with high sweep and Reynolds number by reshaping the wing airfoils to obtain specific pressure distribution characteristics that control the crossflow growth near the leading edge. The CATNLF method also addresses TollmienSchlichting transition, attachment line transition, and Görtler vortices. During the wind tunnel test, data were acquired to address three primary test objectives: validate the CATNLF design method, characterize the NTF laminar flow testing capabilities, and establish best practices for laminar flow wind tunnel testing. The present paper provides both experimental and computational data to understand the CRM-NLF laminar flow characteristics, as well as address the three primary test objectives. The effects of angle of attack and Reynolds number on the CRM-NLF laminar flow extent are studied, and the dominant transition mechanism is evaluated at a variety of test conditions. Critical N-factors are calculated for the NTF environment, and a discussion on best practices for laminar flow wind tunnel testing is provided. The CRM-NLF in the NTF provided initial confirmation of the ability of the CATNLF method to suppress crossflow growth and enable significant extents of laminar flow on transport wings with high sweep and Reynolds numbers.
\end{abstract}

\section{Nomenclature}

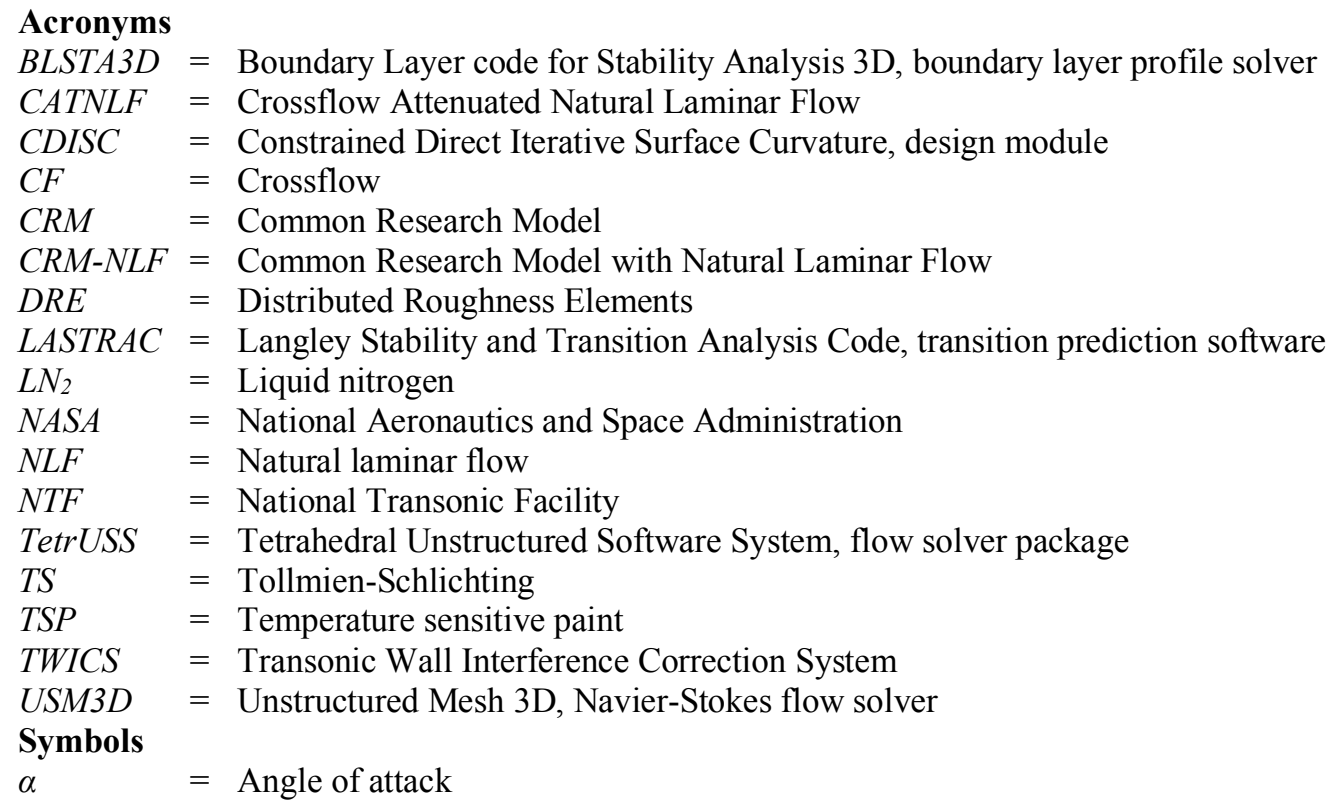

${ }^{1}$ Research Aerospace Engineer, NASA Langley Research Center, Mail Stop 499, Hampton, VA 23681, AIAA Member.

${ }^{2}$ Senior Research Engineer, NASA Langley Research Center, Mail Stop 499, Hampton, VA 23681, AIAA Associate Fellow.

${ }^{3}$ Research Aerospace Engineer, NASA Langley Research Center, Mail Stop 499, Hampton, VA 23681, AIAA Associate Fellow. 


$\begin{array}{ll}c & =\text { Chord length } \\ C_{P} & =\text { Pressure coefficient } \\ M & =\text { Mach number } \\ P_{T} & =\text { Total Pressure } \\ q_{\infty} & =\text { Dynamic pressure } \\ \operatorname{Re}_{\theta} & =\text { Reynolds number based on attachment line boundary layer momentum thickness } \\ \operatorname{Re}_{c} & =\text { Reynolds number based on local chord length } \\ \operatorname{Reft}_{f_{t}} & =\text { Reynolds number based on } 1 \text { foot } \\ \operatorname{Re}_{A C} & =\text { Reynolds number based on mean aerodynamic chord } \\ \operatorname{Re}_{t} & =\text { Reynolds number based on chordwise transition location } \\ (s / c)_{A L} & =\text { Arc length location of the attachment line nondimensionalized by local chord } \\ T_{T} & =\text { Total temperature } \\ x / c & =\text { x-location nondimensionalized by local chord } \\ (x / c)_{t} & =\text { x-location of transition nondimensionalized by local chord } \\ \eta & =\text { Semispan location nondimensionalized by semispan length } \\ \Lambda_{L E} & =\text { Leading-edge sweep }\end{array}$

\section{Introduction}

$\mathrm{T}_{\mathrm{s}}^{\mathrm{H}}$ HE aircraft industry is continually developing new vehicles to accommodate the growing demand for fast and efficient passenger and freight delivery capabilities. For aircraft companies to stay competitive, they must not only have a sound business model, but also continually assess new technologies that could provide an edge in aircraft efficiency and help meet new environmental regulations. One aerodynamic technology that is regarded as having a high potential for future aircraft is laminar flow. Increasing the extents of laminar flow on an aircraft is known to reduce vehicle drag, which reduces the overall fuel burn and offers corresponding reductions in operating costs and emissions.

Increasing the extents of laminar flow on an aircraft is dependent on delaying transition to turbulent flow by controlling disturbances in the laminar boundary layer. One such disturbance is bypass transition, which is related to the surface quality of the component where laminar flow is desired. Considerations to avoid bypass transition must be made in both the manufacturing and design processes of the laminar flow component. Such considerations include manufacturing a smooth surface with minimal gaps and steps, as well as keeping the surface free of imperfections during operation, including ice, paint damage, and insect remains. For the main wing of an aircraft, avoiding steps and gaps typically implies that laminar flow is only targeted for the upper surface to accommodate access panels on the lower surface. Additionally, laminar flow on the upper surface of the wing necessitates that the design of high-lift devices avoids gaps near the leading edge on the upper surface, such as with a Kruger flap or a smooth variable camber leading-edge flap. The Boeing Eco-Demonstrator flight tests assessed the effectiveness of Kruger flaps and surface coatings at minimizing the adverse impact of insect remains acquired at takeoff and landing conditions on laminar flow extent [1]. For components with moderate or no leading-edge sweep, such as nacelles, the primary disturbance that must be controlled to delay transition is the growth of Tollmien-Schlichting (TS) boundary layer instabilities. TS growth can be controlled by tailoring the surface pressure distribution toward a favorable streamwise gradient. Examples of this approach that are currently in service are the laminar flow nacelle on the Boeing 787 MAX [2] and the laminar flow wing on the Honda Jet [3].

Lifting surfaces, such as the wing or tail, offer the greatest potential drag reduction due to laminar flow. These components usually have a leading edge that is swept back to allow the aircraft to fly faster without generating excessive wave drag. Some vehicle concepts have reduced cruise speeds (approximately Mach 0.70) and therefore fairly low sweep values. However, most recent and proposed aircraft, especially those with longer ranges, have cruise Mach numbers closer to 0.85 , which requires the higher sweep values. Increasing sweep to the values of typical transport wings or tails causes a corresponding growth of crossflow (CF) boundary layer instabilities that lead to transition very near the leading edge. Several approaches have been investigated to control the growth of $\mathrm{CF}$ disturbances on swept components. The two primary approaches to date are to reduce the sweep or add a leading-edge flow control system. The reduced sweep naturally reduces CF growth, and was used in the recent European BLADE flight experiment [4], but requires the vehicle to cruise at a slower speed. A leading-edge suction system was the subject of the AFloNext flight experiment [5]. While both approaches have proven effective at suppressing CF growth, inherent penalties are associated with both techniques, including flight time and productivity penalties when reducing sweep, or weight and complexity penalties when adding a suction system. A concept to delay CF transition that would eliminate these penalties is Distributed Roughness Elements (DRE), which appeared promising in some initial wind 
tunnel testing. However, subsequent flight tests gave inconsistent results, and the concept has yet to be proven effective at the higher Reynolds numbers associated with typical transports [6].

A new alternative to delaying transition on highly swept components is the Crossflow Attenuated Natural Laminar Flow (CATNLF) design method, which tailors the wing pressure distribution via airfoil shaping to control both TS and CF disturbances. The method has been applied computationally to both transonic and supersonic transport configurations with significant leading-edge sweep and Reynolds numbers [7, 8]. Recent work has been conducted to experimentally validate the computational predictions of the CATNLF method. A transport wing was designed using the CATNLF method, referred to as the Common Research Model with Natural Laminar Flow (CRM-NLF), [9] and a wind tunnel model was built and tested in the National Transonic Facility (NTF) at the NASA Langley Research Center in 2018. Figure 1 shows the semispan model mounted to the sidewall in the NTF. The wind tunnel test aimed to address three primary objectives:

1. Validate the CATNLF design methodology and analysis tools

2. Characterize the NTF laminar flow testing capabilities

3. Establish best practices for laminar flow wind tunnel testing

Initial results from this test were previously published and indicated that the CATNLF method was successful at suppressing the CF growth near the leading edge [10]. Results also indicated good agreement between the computational predictions and the experimental data regarding pressure distributions and laminar flow extent. Several challenges were encountered while acquiring higher Reynolds number data, which limited the analysis at those conditions.

The previous publication only provided a sample of preliminary data and analysis from the wind tunnel test. The present paper includes additional analysis of the data with the goal of understanding the laminar flow characteristics of the wind tunnel model, as well as providing a more thorough characterization of the NTF environment when possible.

\section{Design and Testing of the CRM-NLF}

The wind tunnel test of the CRM-NLF aimed to experimentally validate the computational predictions of the CATNLF method. Several computational tools and experimental techniques were used in this effort to support the experiment design, operation, and data analysis. This section will briefly cover the design and testing of the CRMNLF, including a highlight of the CATNLF method and the experimental setup. A more detailed description of the design and testing of the CRM-NLF can be found in previous publications $[9,10]$.

\section{A. CATNLF Design Method and Analysis Tools}

The aerodynamic design of the CRM-NLF wind tunnel model utilized the new CATNLF design method and analysis tools. The CATNLF method is built into the CDISC toolset [11], which is comprised of both a design and transition prediction capability, illustrated in the framework shown in Figure 2. This framework was employed for both the design of the model and the analysis of the wind tunnel results. The design of the CRM-NLF utilized both the design and transition prediction loops, while the analysis of the wind tunnel results required only the transition prediction loop. In this subsection, the primary codes used, shown in green boxes in Figure 2, will be briefly described, followed by a summary of the CATNLF design approach.

\section{Computational Tools}

The flow solver selected for the design and analysis of the CRM-NLF was USM3D, which is part of the TetrUSS software system [12]. The code solves the Reynold Averaged Navier-Stokes equations using a triangulated surface grid with tetrahedral cells for the volume grid. The solver uses a cell-centered, upwind scheme, with turbulent closure obtained using the Spalart-Allmaras turbulence model for all the cases shown in this paper. USM3D has a forced laminarization capability to model regions of laminar flow, which was used for the design of the CRM-NLF.

The surface pressure results from the flow solver are used by the transition prediction module to determine the boundary layer instability characteristics for laminar flow cases. The LASTRAC code [13] was used to perform the boundary layer stability analysis for both the design of the CRM-NLF and the analysis of wind tunnel results. For this work, the $\mathrm{e}^{\mathrm{N}}$ method with linear stability theory was used, including effects of compressibility but not curvature. The fixed-beta method was used for all calculations and only stationary CF and TS disturbances were considered.

To perform the stability analysis, LASTRAC needs the boundary layer mean flow profiles at designated locations. These boundary layer profiles could have been extracted directly from the flow solver results, however, this approach requires a much larger grid with approximately 3 times as many grid cells in the boundary layer. To save computational resources, especially for design cases, an alternative approach was used. The BLSTA3D code [14] was utilized to 
calculate the required boundary layer mean flow profiles from the flow solver surface pressure results. For this work, the conical flow option was used to account for the sweep and taper of the CRM-NLF wing. BLSTA3D also calculated the attachment line $\operatorname{Re}_{\theta}$ values, which were used to predict the state of the attachment line boundary layer.

\section{CATNLF Design Method}

The CATNLF design method enables laminar flow by controlling both CF and TS growth through shaping the airfoil to obtain specific pressure distribution characteristics. As mentioned, this strategy is built into the CDISC design module, which is a knowledge-based design tool that automatically generates target pressure distributions according to specified design requirements. CDISC alters the geometry according to prescribed sensitivity derivatives to change the geometry to drive the flow solver analysis pressures toward the target pressure distributions, while meeting other flow and geometry constraints.

The CATNLF method is based on specific pressure distribution characteristics, illustrated in Figure 3. It consists of a rapid flow acceleration very near the leading edge terminating in a sudden turn to a fairly flat rooftop. The location of the sudden turn is determined internally within CDISC to limit the CF growth below a specified N-factor level. The relatively flat pressure gradient aft of the sudden turn is also determined within CDISC to control TS growth so that it increases gradually and reaches the specified TS critical N-factor level at the desired transition location.

The CRM-NLF was designed to be representative of a generic transonic transport. The baseline configuration was the Common Research Model (CRM), which is an open-geometry transport that has been the subject of several computational and experimental investigations worldwide [15]. The CRM-NLF was designed for cruise conditions of Mach 0.85 , lift coefficient of 0.50 , and mean aerodynamic chord Reynolds number of 30 million. The design was performed using estimated laminar flow extents in a flight environment, which was modeled by using a critical Nfactor level of 10 for both TS and CF. The primary design objective for the CRM-NLF was to obtain as much laminar flow as possible, so the desired transition location was prescribed to be just upstream of the shock, even if that choice resulted in a stronger shock.

In addition to the pressure architecture aspects used to control CF and TS growth on the CRM-NLF, the model needed to address transition due to Görtler vortices and a turbulent attachment line. Curvature constraints were imposed using CDISC to avoid any concavity in regions of laminar flow to address transition due to Görtler vortices. The design technique employed to address attachment line transition was to locally reduce the attachment line $\operatorname{Re}_{\theta}$ values inboard according to Poll's criteria for relaminarization (i.e., $\operatorname{Re}_{\theta}<100$ ) [16]. This was needed to avoid attachment line contamination that occurs on swept wings from the turbulent fuselage boundary layer. On the CRM$\mathrm{NLF}$, the local reduction in $\mathrm{Re}_{\theta}$ values was obtained by reducing the leading-edge sweep over the inboard $10 \%$ of the wing. Outboard of this reduced leading-edge sweep section, the $\operatorname{Re}_{\theta}$ values remained below 235 to avoid attachment line transition. Additional information on the design of the CRM-NLF model can be found in a previous publication [9].

\section{B. Experimental Setup}

The CRM-NLF wind tunnel model was tested in the National Transonic Facility (NTF) at the NASA Langley Research Center in 2018. The NTF is the world's largest fan-driven, closed-circuit, continuous-flow, pressurized wind tunnel and is capable of operating either in dry air at warm temperatures or in nitrogen gas from warm to cryogenic temperatures $[17,18]$. The facility enables testing of full-span or semispan models from subsonic to transonic speeds at Reynolds numbers up to full-scale flight values. The NTF was selected for the CRM-NLF experiment based on its high Reynolds number capability and acceptably low turbulence levels, which provide a relevant environment for laminar flow testing. Flow quality measurements previously acquired in the NTF [19], along with previous natural laminar flow (NLF) testing by Crouch et al. [20], estimated turbulence intensity in the test section to be approximately $0.24 \%$, which was concluded to be acceptable for laminar flow testing in the absence of bypass transition.

The test article used in this experimental investigation was a 5.2\% scale semispan model of the CRM-NLF. The wing was instrumented with 230 static pressure ports (140 on the upper surface and 90 on the lower surface) to ensure the CATNLF pressure distribution characteristics discussed previously were obtained on the wind tunnel model. The pressure ports were arranged along nine streamwise rows across the wing semispan shown in Figure 4, with leadingedge pressure ports only present on 4 of those rows to reduce the bypass transition that occurs from leading-edge pressure ports. Table 1 lists the model-scale reference parameters for the CRM-NLF model. The majority of experimental data were acquired at a nominal Mach number of 0.86 , as this condition correlated best to the computational results from the design condition of Mach 0.85 . The focus on the test was on near-cruise angles of attack, at a variety of temperature and pressure combinations to vary mean aerodynamic chord Reynolds numbers from 10 to 30 million. The primary nominal test conditions are listed in Table 2. 
Table 1. Reference parameters for the CRM-NLF model.

\begin{tabular}{|l|l|}
\hline \multicolumn{2}{|c|}{ Model-Scale Reference Parameters } \\
\hline Reference Area & 5.584 sq.ft. \\
\hline Reference Chord & 14.342 in. \\
\hline Semispan Length & 60.151 in. \\
\hline Leading-Edge Sweep (Outboard of Break) & $37.3 \mathrm{deg}$. \\
\hline
\end{tabular}

Table 2. Summary of the primary nominal test conditions.

\begin{tabular}{|c|c|c|c|c|c|c|}
\hline \multicolumn{7}{|c|}{ Primary Nominal Test Conditions } \\
\hline Mach & $\boldsymbol{\alpha}$ (deg.) & $\mathbf{T}_{\mathbf{t}}\left({ }^{\circ} \mathbf{F}\right)$ & $\mathbf{P}_{\mathbf{t}}(\mathbf{p s i a})$ & $\mathbf{q}_{\infty}(\mathbf{p s f a})$ & $\mathbf{R e}_{\mathbf{f t}}(\mathbf{x 1 0})$ & $\mathbf{R e}_{\mathbf{M A C}}(\mathbf{x 1 0})$ \\
\hline \multirow{3}{*}{0.86} & \multirow{3}{*}{1.5 to 3.0} & -40 & 26 to 39 & 1180 to 1780 & 8.4 to 12.6 & 10.0 to 15.0 \\
\cline { 3 - 7 } & & -50 & 24 to 39 & 1120 to 1800 & 10.5 to 16.7 & 12.5 to 20.0 \\
\cline { 3 - 7 } & & -150 & 26 to 39 & 1200 to 1800 & 16.7 to 25.1 & 20.0 to 30.0 \\
\hline
\end{tabular}

The data acquired during the CRM-NLF test included: force and moment, surface static pressure, model deformation, and transition visualization data. The surface static pressure and transition visualization data are used to validate the CATNLF design method and characterize the NTF, and are the primary results presented in this report. A previous publication documented the force and moment data acquired during the test [21]. The data presented in this paper have been conditionally sampled and corrected utilizing the Transonic Wall Interference Correction System (TWICS) [22]. The CRM-NLF geometry and some wind tunnel data will be released to the public on the CRM website at https://commonresearchmodel.larc.nasa.gov/crm-nlf/.

The CRM-NLF wing was painted with Temperature Sensitive Paint (TSP) to visualize regions of laminar and turbulent flow in the wind tunnel. TSP shows regions of laminar flow when a temperature gradient is introduced because of the temperature difference on the surface between the laminar regions with lower heat transfer rate and the turbulent regions with higher heat transfer rate [23]. During the CRM-NLF test, two mechanisms of introducing the required temperature gradient were investigated: rapidly cooling the freestream flow via a rapid liquid nitrogen (LN $\left.\mathrm{L}_{2}\right)$ injection and heating the wing surface via a carbon-based resistive heating layer. The rapid $\mathrm{LN}_{2}$ injection method has been used previously in cryogenic facilities for TSP imaging, but has several drawbacks, including altering the tunnel conditions during data acquisition, increasing the total consumption of $\mathrm{LN}_{2}$, and requiring additional time to acquire each data point. The carbon-based heating layer is a newly developed technology that provides resistive heating via a conductive paint layer beneath the TSP [24, 25]. The heating layer enables stable tunnel conditions during data acquisition and reduces $\mathrm{LN}_{2}$ consumption, thus providing significant data quality improvements and cost saving over the $\mathrm{LN}_{2}$ injection method. Details on the development and application of this carbon-based resistive heating layer used during the CRM-NLF test can be found in previously published reports [10, 26].

The TSP images provide the experimental transition visualization needed to assess the CATNLF method. An example of a TSP image is shown in Figure 5 with several features noted on the image. The light regions on the image are laminar and the darker regions are turbulent. Bypass transition appears as a turbulent wedge emanating at or near the leading edge, an example of which is labeled on the image. At model scale, bypass transition is typically caused by very small surface imperfections, such as pressure ports, particulates in the tunnel, or paint damage. If the bypass transition becomes too frequent, the turbulent wedges can coalesce ahead of the natural transition front such that natural transition can no longer be detected experimentally at that condition. Bypass transition is typically more frequent at higher Reynolds numbers when the boundary layer is thinner, and is discussed in more detail in the following section of this paper. Natural transition is identified as the straight boundary of varying chord locations between the laminar and turbulent regions, an example of which is labeled on the image. For this analysis, all natural transition fronts are determined manually by visual inspection of each TSP image. If a natural transition front can be seen on both sides of a turbulent wedge, it is assumed that the natural transition would have occurred at that front in the absence of bypass transition, and a straight line connecting the front through the wedge is drawn. However, if turbulent wedges coalesce forward of any straight front, the only conclusion that can be made in that region is that natural transition is at least as far aft as the coalescence location. The natural transition location is of particular interest to address the test goals of validating the CATNLF method and characterizing the NTF.

In addition to the TSP images, the experimental static pressure data are central to assessing the original test objectives. The static pressure data acquired provide insight into how well the predicted CATNLF pressure distributions were obtained during testing. However, the limited distribution of pressure ports prohibits performing stability analysis calculations directly on experimental pressure data. In the previous publication with preliminary results, an infilling method was applied to the uncorrected experimental data using the computational flow solution 
results, and Mach and angle-of-attack shifts were required for adequate agreement [10]. In this report, a new flow solution was obtained for every point analyzed on a new grid at conditions that match the corrected wind tunnel conditions. These new flow solutions agree well with the experimental data at corresponding conditions with no Mach or angle-of-attack shift required. An example of the type of agreement between computational and experimental pressures is shown in Figure 6. The overall agreement is of sufficient quality to enable use of the computational pressures in all stability analysis calculations in this report. Improved capabilities of infilling the experimental data are being pursued for future work to ensure the stability analysis calculations most accurately represent the TSP images acquired.

\section{Transition Characteristics of the CRM-NLF in the National Transonic Facility}

The wind tunnel test completed in the NTF acquired data to enable in-depth analysis of the transition characteristics of the CRM-NLF, including surface pressure data and transition visualization images. Additional computational results, such as flow solutions and boundary layer stability analysis calculations, have been obtained at conditions that match those at which experimental data were acquired. This section will include several computational and experimental results that aim to provide insight into the dominant transition mechanisms on the CRM-NLF in the wind tunnel environment.

\section{A. Observations Regarding Extents of Laminar Flow}

During the wind tunnel test, conditions were selected such that the acquired data could be used to determine trends in the laminar flow extents. The two primary factors on extent of laminar flow being analyzed in this paper are angle of attack and Reynolds number. Secondary factors relating to experimental technique, including temperature gradient mechanism and dynamic pressure, are briefly investigated as well. This subsection includes general observations regarding how each factor affects the extent of laminar flow seen in the wind tunnel. The effects of each factor on the growth of CF and TS are also included, however, any discussion on the mechanism responsible for transition is held for the following subsection.

\section{Angle-of-Attack Effects on Laminar Flow Extent}

The angle-of-attack range over which the majority of experimental data were acquired focused on near-cruise angles from $1.5-3.0$ degrees in 0.5 degree increments. To isolate the effect of angle of attack on laminar flow extent, observations are made on data sets where all other parameters, such as Reynolds number, are held constant. For the analysis in this paper, the angle-of-attack effect is studied at a mean aerodynamic chord Reynolds number of 15 million because it is the highest Reynolds number that produced high-quality transition visualization images during testing (i.e., moderate bypass transition such that natural transition fronts can still be determined across the span). The 15 million mean aerodynamic chord Reynolds number cases used in this assessment were obtained at a tunnel total temperature of $+40^{\circ} \mathrm{F}$.

The transition visualization images from the angle-of-attack sweep can be seen in Figure 7. At each angle of attack, significant regions of laminar flow can clearly be seen. Each of the 4 images show 23 turbulent wedges emanating at or near the leading edge. Most of these turbulent wedges are seen in the same location in every image, which suggests many of these surface imperfections are likely permanent defects in the paint surface, and that new surface imperfections are not rapidly accumulating during the polar from which these images were taken. The relatively moderate amount of bypass transition seen in these images allows a natural transition front to be determined at each angle of attack.

The experimental transition fronts are approximated from each image using the method described in the previous Experimental Setup section. Figure 8 shows these experimental transition fronts for all 4 conditions overlaid for direct comparison. The CRM-NLF in the NTF shows a gradual decrease in total surface area of laminar flow with increased angles of attack. Based on these approximated experimental fronts, the percent surface area of laminar flow for angles of attack of 1.5, 2.0, 2.5, and 3.0 degrees are approximately $42 \%, 37 \%, 30 \%$, and $30 \%$, respectively. The transition fronts show that, over the inboard portion of the wing, higher angles of attack transition further forward compared to the lower angles of attack, which contribute to the decreased surface area of laminar flow at the higher angles of attack. At the lower angles of attack, a discontinuity in the transition front appears in the outboard portion of the wing, where a sudden increase in chordwise laminar flow extent occurs. This discontinuity is not apparent at the higher angles of attack. The varying extents of laminar flow are better understood through the surface pressure data acquired at each angle of attack. Figure 9 investigates the sudden increase in chordwise extent of laminar flow that is seen outboard between Rows $\mathrm{F}$ and $\mathrm{G}$ at the 1.5 degree case. The pressure distributions in this figure show a collapse of the rooftop pressures at Row F, causing a sudden increase in pressure (shock) forward on the chord corresponding to the more 
forward transition location at these stations. Further outboard at Row G, the rooftop pressures are no longer collapsing, thereby eliminating the forward shock, thus, moving the transition location significantly further aft on the chord. This collapse of pressures leading to a double-shock pressure distribution is only observed at the lower angles of attack, which provides an explanation as to why the discontinuity in the transition front only occurs at the lower angles of attack.

In addition to studying the experimental transition fronts, the effect of angle of attack on laminar flow extent can be further characterized using computational results. Several stability analysis calculations were performed on computational pressures at conditions that matched the corrected wind tunnel data. These calculations enable an evaluation of how angle of attack influences both CF and TS growth. An example of how the TS and CF growth changes with progression out the span of the CRM-NLF wing is shown in Figure 10. This example figure is from an angle of attack of 2.5 degrees, but the three other angles of attack show similar trends. It can be seen that the TS growth is strongest at the inboard station (Row A), and decreases with progression out the span. In the CF growth plot, the most inboard station (Row A) has the lowest CF N-Factor growth because that station is located on the reduced leading-edge sweep region of the wing. CF growth is known to be strongly dependent on leading-edge sweep, where lower sweeps produce significantly less CF growth than higher sweeps. Outboard of Row A is the constant leading-edge sweep region of the wing (Rows B $-\mathrm{I}$ have $\Lambda_{\mathrm{LE}}=37.3 \mathrm{deg}$.), and CF follows a similar trend as the TS growth, where the highest CF growth is seen inboard and gradually decreases out the span. This reduction in both TS and CF growth out the span of the wing is likely a function of the reduction of chord Reynolds number from inboard to outboard. The N-Factor growth for all 4 angles of attack at several stations are shown in Figure 11. Consistent trends with angle of attack can be seen across the wing. The TS N-Factor plots show that increasing angle of attack increases TS growth. This increased TS growth at the higher angles of attack provides one possible explanation as to why the higher angles of attack showed slightly smaller experimental extents of laminar flow. The chord Reynolds numbers at each spanwise location are identical for all angles of attack, so the increase in TS with angle of attack is likely due to changes in pressure gradients. Figure 12 shows an example of the pressure distribution changes with angle of attack at one station (Row E). This image shows the more favorable pressure gradients on the upper surface at the lower angles of attack, which are known to reduce TS growth. Trends can also be observed on the CF growth with changing angle of attack. The $\mathrm{CF}$ growth can be categorized in two regions: rapid growth near the leading edge (referred to as "peak CF" in this paper) and more gradual midchord growth. Figure 11 shows that the maximum peak $\mathrm{CF}$ is increased very slightly with increasing angle of attack, however, the effect is minimal with all peak CF N-factors within less than $1 \mathrm{~N}$-Factor of each other. For comparison, Figure 10 shows that the maximum peak CF out the span varies by approximately $2.5-3 \mathrm{~N}$-Factor. The maximum peak CF has a strong dependence on Reynolds number, and for the angle-of-attack sweep all chord Reynolds numbers are identical, which provides an explanation as to why the variation in peak $\mathrm{CF}$ with angle-of-attack is minimal. However, significant changes to the character of midchord $\mathrm{CF}$ growth can be seen with angle of attack. The lower angles of attack show significant growth of midchord CF aft of the peak, while the higher angles of attack do not. This midchord CF growth is highly dependent on the pressure distribution, where the more favorable pressure gradients seen at the lower angles of attack (example shown in Figure 12) can cause CF to grow. These observations on how angle of attack affects laminar flow (both experimental extent and computational CF and TS growth) aid in understanding the CRM-NLF in the NTF.

\section{Reynolds Number Effects on Laminar Flow Extent}

The second factor on laminar flow extent studied in this paper is Reynolds number. In the previous subsection, the angle-of-attack data used for analysis were acquired in a single polar at constant tunnel conditions, which isolates the effect of angle of attack. However, the effect of Reynolds number is harder to characterize because it is not possible to hold all other experimental parameters constant in a Reynolds number sweep. In order to vary Reynolds number in the NTF, the total temperature and/or dynamic pressure is altered. One additional challenge with Reynolds number is the increased occurrence of bypass transition at higher Reynolds numbers. As the Reynolds number increases, the boundary layer thins, which results in smaller surface imperfections reaching the critical height and causing bypass transition. It was observed that additional turbulent wedges often accumulated during testing, and a model access was required to sand and polish the paint surface to remove the defects. This means that transition images acquired directly after a model access will likely show less bypass transition than those taken after several data points, resulting in images that may not follow the trend of increased wedges at higher Reynolds number. Images with severe bypass transition prohibit determining the natural transition front because the turbulent wedges coalesce forward of the natural transition location. Table 3 shows relevant parameters for the experimental data points used in this paper to study Reynolds number effect. The Reynolds number sweep used an angle of attack of 1.5 degrees because this angle provided the best transition images across the Reynolds number range. This is likely due, in part, to 1.5 degrees being the first angle of attack in the polar and it therefore had the fewest data points since the previous paint polish. 
Table 3. Parameters relevant to laminar flow for the transition visualization images used in the Reynolds number sweep.

\begin{tabular}{|c|c|c|c|c|c|c|c|}
\hline \multicolumn{7}{|c|}{ Image Quality Parameters for Reynolds Number Sweep TSP Images } \\
\hline $\begin{array}{c}\mathbf{R e}_{\mathbf{M A C}} \\
(\mathbf{m i l l i o n s})\end{array}$ & $\begin{array}{c}\boldsymbol{\alpha} \\
\text { (degrees) }\end{array}$ & $\begin{array}{c}\mathbf{T}_{\mathbf{T}} \\
\left({ }^{\circ} \mathbf{F}\right)\end{array}$ & $\begin{array}{c}\mathbf{q}_{\infty} \\
(\mathbf{p s f a})\end{array}$ & $\begin{array}{c}\text { Bypass } \\
\text { Transition } \\
\text { Level }\end{array}$ & $\begin{array}{c}\text { Number of } \\
\text { Turbulent } \\
\text { Wedges }\end{array}$ & $\begin{array}{c}\text { Data Points } \\
\text { Since } \\
\text { Paint Polish }\end{array}$ & $\begin{array}{c}\text { Laminar Flow } \\
\text { Surface Area }\end{array}$ \\
\hline 10.0 & 1.5 & +40 & 1177 & Minimal & 17 & 17 & $44 \%$ \\
\hline 12.5 & 1.5 & +40 & 1473 & Moderate & 27 & 9 & $42 \%$ \\
\hline 15.0 & 1.5 & +40 & 1768 & Moderate & 23 & 1 & $42 \%$ \\
\hline 17.5 & 1.5 & -53 & 1563 & Severe & 65 & 9 & $22 \% * *$ \\
\hline 20.0 & 1.5 & -53 & 1787 & Severe & $36^{*}$ & 1 & $12 \% * *$ \\
\hline 22.5 & 1.5 & -153 & 1354 & Severe & $33^{*}$ & 9 & $6 \% * *$ \\
\hline
\end{tabular}

*Leading-edge transition is present, reducing wedge count because no wedges can be seen in that region

**Natural transition fronts are very limited or not visible due to severity of bypass transition

The transition images from the Reynolds number sweep are shown in Figure 13. Significant extents of laminar flow can be seen at Reynolds numbers less than or equal to 15 million, where clear natural transition fronts can be determined across most of the span. Beginning at a Reynolds number of 17.5 million, the regions where a natural transition front can be determined are significantly reduced due to turbulent wedges coalescing. This reduction in natural transition is one possible explanation for the sudden decrease in percent surface area of laminar flow (seen in Table 3) that occurs at the 17.5 million condition. Another cause of the loss of laminar flow surface area is due to the leading-edge transition at the 20.0 and 22.5 million conditions. This leading-edge transition can be due to either peak $\mathrm{CF}$ growth or bypass transition of the attachment line, and is further investigated in the following Assessment of Transition Mechanisms subsection. The limited natural transition visible at and above 17.5 million makes characterizing the CRM-NLF at these conditions challenging. The approximated experimental transition fronts for all six Reynolds numbers are shown in Figure 14. The 17.5 - 22.5 million cases are bypass transition fronts. It can be assumed that these bypass transition fronts would be further aft in the absence of bypass transition. The three lower Reynolds numbers where natural transition can be detected show very similar transition fronts across the span. This suggests that the transition location in this region is shock-limited at these conditions, otherwise the transition front is expected to move forward with increased Reynolds number.

Computational results can be used to characterize the Reynolds number effect on laminar flow even at the conditions that proved challenging in the wind tunnel. Figure 15 has the TS and CF N-factor growth at several stations along the wing at all six Reynolds numbers. The $\mathrm{N}$-factor growth plots show that both TS and CF increase relatively uniformly with increased Reynolds numbers across the span. The TS growth appears to scale roughly with the square root of Reynolds number, whereas the CF appears to scale directly with Reynolds number. The character of $\mathrm{N}$-factor growth at each station remains nearly unaltered. The peak CF growth at this angle of attack varies by $3-4 \mathrm{~N}$-factor from 10.0 to 22.5 million Reynolds number. The Reynolds number trends seen in the computational results can be used to assess dominant transition mechanisms on the CRM-NLF, which is discussed in the following subsection.

\section{Experimental Technique Effects on Laminar Flow Extent}

In addition to the aerodynamic factors on laminar flow extent previously discussed, the experimental technique can also influence the laminar flow extent. Two such experimental techniques are the method of obtaining the temperature step required for TSP images and the tunnel conditions (i.e., combination of temperature and dynamic pressure) used to obtain the desired Reynolds number.

As previously mentioned in the Experimental Setup section, the TSP images require a temperature gradient to visualize the laminar flow. During testing, there were two mechanisms to provide the required temperature gradient: a rapid $\mathrm{LN}_{2}$ injection and a carbon-based resistive heating layer. The liquid nitrogen injection cooled the freestream flow, while the carbon-based heating layer heated the model wing. Altering surface temperature is known to potentially alter transition location, so a brief investigation into the effects of the temperature gradient mechanism is included in this paper. Figure 16 shows an example of both temperature gradient mechanisms at the same tunnel conditions. The images are from consecutive runs, so no changes to the paint surface were made between the two points. The $\mathrm{LN}_{2}$ injection image was acquired after the heating layer image, which can explain the increase in bypass transition seen in the $\mathrm{LN}_{2}$ injection image. The $\mathrm{LN}_{2}$ injection causes the Reynolds number to increase during data acquisition, which also increases the likelihood of bypass transition. The $\mathrm{LN}_{2}$ injection method produced a $9{ }^{\circ} \mathrm{F}$ reduction in the freestream temperature, while the heating layer method produces a $5-10^{\circ} \mathrm{F}$ increase in model surface temperature across the wing. Figure 17 compares the approximate experimental transition front from both images, and 
shows good agreement between the two images. The midspan region shows the liquid nitrogen injection transition location further forward, which is due to the excess bypass transition in that region. The other angles of attack showed similar agreement between the two temperature gradient mechanisms. At the level of accuracy possible in determining transition location from these experimental images, it appears the transition gradient mechanism does not have a significant effect on the natural transition location.

The second experimental technique that may influence the laminar flow extent is the tunnel conditions at which the data are acquired. The NTF can vary temperature and pressure independently, which means that the same Reynolds number can be acquired at two different temperatures by altering pressure. During the test, there was some overlap in Reynolds numbers at each temperature, which provides an opportunity to assess if the tunnel conditions had a noticeable impact on laminar flow extent. One concern is that different temperature/pressure combinations could impact laminar flow by changing model deformation at the different dynamic pressures. To investigate this, two data points are studied from the 12.5 million Reynolds number and 1.5 degrees angle-of-attack nominal condition that were acquired at different tunnel temperature/pressure combinations. The two transition images are shown in Figure 18; Figure 18a was acquired at a total temperature of $+40^{\circ} \mathrm{F}$ and dynamic pressure of $1473 \mathrm{psfa}$, and Figure $18 \mathrm{~b}$ was acquired at a total temperature of $-53^{\circ} \mathrm{F}$ and dynamic pressure of 1115 psfa. Qualitatively, the images appear very similar, with only a slight increase in bypass transition seen in the $-53^{\circ} \mathrm{F}$ case. Figure 19 compares the transition fronts from each image, and shows the transition location agrees well across the span, especially outboard, where any differences in static aeroelastic deflection due to different dynamic pressures would be most evident. The inboard mismatch is primarily due to wedges coalescing at the $-53^{\circ} \mathrm{F}$ case. The surface pressure data (not shown) indicated little to no change between the two points at all 9 pressure rows. This suggests that the change in model deflection with dynamic pressure is relatively small and likely has little effect on the laminar flow.

\section{B. Assessment of Transition Mechanisms}

Several mechanisms of transition were possible on the CRM-NLF in the NTF, including CF, TS, bypass, and attachment line transition. This subsection discusses the transition mechanisms observed during the wind tunnel test and some of the challenges encountered in this analysis. Transition is highly dependent on the environment, so many of the findings presented in this paper are specific to the NTF, and some conclusions are expected to be different if the CRM-NLF were in a flight environment. Experimental and computational results are used together to identify which mechanism is responsible for transition. The transition images give insight into both bypass and attachment line transitions, and the computational $\mathrm{N}$-factor growth is used with the experimental transition location for identifying both CF and TS transitions.

Bypass transition on the upper surface of the wing has been previously discussed, and is identified in the images by the presence of a turbulent wedge. When the wedges become too frequent and coalesce, most commonly at the higher Reynolds number conditions, the bypass transition prohibits determining the natural transition location. An additional challenge with bypass transition occurs when the surface imperfection is located on the attachment line of the wing. Bypass transition of the attachment line can lead to significant regions of the wing that show leading-edge transition. This phenomenon was discussed in a previous paper published on the CRM-NLF wind tunnel test [10]. The most effective way to identify bypass attachment line transition is with two TSP images acquired at the same conditions, one with leading-edge transition and one without. An example set of images can be seen in Figure 20. CF transition can also lead to leading-edge transition over a similar region of the wing, however, since an image exists at the same conditions with full-span laminar flow (i.e., no region of leading-edge transition), it can be deduced that the leadingedge transition is not due to $\mathrm{CF}$ as that should always be present at a given condition. Bypass transition can be more inconstant; it can occur during testing if there is a new surface imperfection acquired, or be removed after polishing the paint surface. As mentioned in the previous section, the CRM-NLF was designed to protect against attachment line contamination with the reduced sweep inboard. Outboard of the reduced sweep, the attachment line was often above a $\operatorname{Re}_{\theta}$ value of 100 , which means the attachment line state is dependent on the inboard state. The design concept was that the attachment line boundary layer relaminarizes over the reduced sweep section, and would remain laminar out the span. This design concept proved effective at many conditions. However, if attachment line bypass transition occurs, the attachment line remains turbulent until the $\operatorname{Re}_{\theta}$ value reduces to below 100 , often significantly outboard. This theory is supported by correlating the computational values of $\operatorname{Re}_{\theta}$ with the attachment line transition and relaminarization semispan locations. An example of this is shown in Figure 21, where it can be seen that the attachment line boundary layer relaminarization occurs at an $\operatorname{Re}_{\theta}$ value of approximately 100 . Once attachment line bypass transition occurs at a given condition, the paint surface must be polished to remove the imperfection causing transition. However, changing conditions can potentially shift the attachment point away from the surface imperfection such that the attachment line bypass transition no longer occurs. Figure 22 shows the computationally predicted attachment location (measured based on nondimentional arc length) for both the angle-of-attack and Reynolds number sweeps. 
These plots illustrate that the attachment line location is primarily related to the angle of attack. It can be assumed that if attachment line bypass transition occurs at a given condition, data acquired at the same angle of attack and higher Reynolds numbers would also show attachment line bypass transition until a paint polish is performed. There were images acquired during a single polar that showed attachment line transition at only some angles of attack, suggesting the attachment line had shifted away from the critical surface imperfection by changing angle of attack.

In the absence of bypass and attachment line transitions, the CRM-NLF wing is expected to transition due to CF and TS. The computational stability analysis results can be used to identify which instability is critical along the wing at different conditions. The transition location is determined from the experimental images, and that transition location is correlated to the stability analysis. An example of a TS-dominant transition can be seen in Figure 23 from Row D at 15 million Reynolds number and 3.0 degrees angle of attack. The $\mathrm{CF}$ N-factors grow rapidly at the leading edge, then are reduced steadily until no more CF growth exists. The TS shows continued growth from the leading edge. The experimental transition location is noted on the plot, and it can be seen that the TS growth has reached an N-factor of 5.5 at the observed transition location. An example of CF transition can be seen in Figure 24 from Row H at 22.5 million Reynolds number and 1.5 degrees angle of attack. At this higher Reynolds number condition, the natural transition location was not observed experimentally due to bypass transition as previously discussed. The wedges coalesced at $\mathrm{x} / \mathrm{c}=0.25$ at this station, so the only conclusion about transition location at this condition is that natural transition would occur at an $\mathrm{x} / \mathrm{c}>0.25$. This information can sometimes still be used to distinguish between TS and $\mathrm{CF}$ transition. The TS growth at this station reaches a maximum of approximately $2.0 \mathrm{~N}$-factor, which is far below the expected critical $\mathrm{N}$-factor in the tunnel. The $\mathrm{CF}$ has the rapid growth near the leading edge, and is followed by midchord growth. At the location of wedge coalescence, the CF N-factor is approximately 5.9. The character of the $\mathrm{CF}$ and TS suggest that this station would likely transition due to midchord CF growth in the absence of bypass transition.

At the higher Reynolds numbers, the CRM-NLF could transition due to the leading-edge CF growth, rather than the midchord CF grown seen in the previous example. The challenge with identifying the leading-edge $\mathrm{CF}$ transition in the experimental images is that it is expected to look very similar to the bypass attachment line transition, where transition occurs at the leading-edge over a midspan region of the wing. For Reynolds numbers greater than or equal to 20 million, there were no TSP images acquired during testing that did not have leading-edge transition. That could mean that bypass attachment line transition always occurred (i.e., a critical-size surface imperfection was present that could not be removed with paint polishing), or it could be due to leading-edge CF transition, which is expected to be consistently present at a given condition. In an attempt to distinguish between bypass attachment line transition and leading-edge $\mathrm{CF}$ transition, the semispan location of transition and relaminarization are correlated to both $\operatorname{Re}_{\theta}$ values and peak CF N-factor values. The leading-edge peak CF $\mathrm{N}$-factor values are taken from the $\mathrm{N}$-factor plots shown in Figures 11 and 15 and are plotted against the semispan location in Figure 25. The trends discussed in the previous subsection in regard to peak CF changes are illustrated in Figure 25, namely that the variation in peak CF values is much more dependent on Reynolds number than angle of attack. Figure $25 \mathrm{~b}$ can be used to predict which regions of the wings could show leading-edge CF transition. For the purposes of this explanation, assume a critical N-factor of 5. Reynolds numbers 17.5 million and below never reach a value of 5, so no leading-edge transition due to CF would be expected at these Reynolds numbers. At the 20.0 million condition, the peak CF N-factors exceed 5 between a semispan location of 0.19 and 0.34 , so leading-edge transition would be expected in that region. As the Reynolds number is increased to 22.5 million, the region where the peak CF N-factors exceed 5 is expanded to be between 0.18 and 0.82 , so the region of leading-edge transition would be expected to increase as well and correspond to those semispan locations. The region of leading-edge $\mathrm{CF}$ transition is dependent on the critical $\mathrm{N}$-factor value in the wind tunnel, which is discussed toward the end of this subsection. The semispan variation of attachment line $\operatorname{Re} \theta$ values are shown in Figure 26 for both the angle-of-attack and Reynolds number sweeps. For these plots, the $\operatorname{Re}_{\theta}$ value of significance is 100 , because any location where $\operatorname{Re}_{\theta}$ is greater than 100 is susceptible to attachment line bypass transition. For conditions where every image showed leading-edge transition, the peak CF variation and the attachment line $\operatorname{Re}_{\theta}$ variation across the semispan can be correlated to the semispan location of leading-edge transition and relaminarization. Figure 27 shows an example of this type of analysis for the 22.5 million Reynolds number condition. Although the figure shows somewhat inconsistent results, it suggests the leading-edge transition may be due to leading-edge $\mathrm{CF}$ growth rather than attachment line transition at this condition. This is deduced from the fact that the $\operatorname{Re}_{\theta}$ values are well below the 100 relaminarization criterion at the outboard location of the leading-edge transition region. The $\mathrm{CF} \mathrm{N}$-factor values remain above 5 within the leading-edge transition region, and is lower on either side, suggesting the $\mathrm{CF}$ critical $\mathrm{N}$-factor at this condition may be around 5 . Thus far, efforts using this method to determine the source of leading-edge transition at higher Reynolds numbers have provided inconsistent results and further investigation is needed to fully understand the leading-edge transition observed in the experiment. Some potential sources of inconsistency are related to the accuracy of determining the experimental spanwise 
transition/relaminarization locations, accuracy of computational pressures and CF N-factor in the leading-edge region, and accuracy of calculated $\mathrm{Re}_{\theta}$ values and Poll's criterion of 100 for relaminarization.

When performing the data analysis to understand the transition mechanisms on the CRM-NLF, an inconsistency between the computational stability analyses and experimental transition locations was observed at several outboard locations on the wing. For a given pressure row, the experimental transition locations from the TSP image was often further aft than the stability analysis codes would run. Figure 28 shows an example of this phenomenon, where the experimental transition location was at approximately $\mathrm{x} / \mathrm{c}=0.50$, but the stability analysis calculations only ran to $\mathrm{x} / \mathrm{c}$ $=0.43$. The stability analysis calculations will terminate automatically when the boundary layer code detects either laminar separation or a very strong pressure gradient (shock). The pressures from this example are shown in Figure 29 , where it can be seen that a strong pressure gradient exists at approximately $\mathrm{x} / \mathrm{c}=0.45$, which explains why the stability analysis calculations terminate near that chordwise location. To understand how the TSP image shows transition aft of this pressure gradient, all experimental pressure recordings during data acquisition (referred to as "frame" data) is investigated. Figure 30 shows all of the frame data from data acquisition, highlighting the large pressure changes between $0.40<\mathrm{x} / \mathrm{c}<0.70$. The minimum and maximum pressures at each pressure port in the shock region can be correlated to pressure distributions from individual frames at different times during the data acquisition, shown in Figure 31. These individual frame pressure distributions show that the minimum and maximum pressure tap values can be explained by the rooftop pressures collapsing. The averaged data fall between these two pressure distributions. The dynamic nature of these pressure distributions seen in the variation of shock location suggest that the standard method of averaging the pressure tap readings to determine the experimental pressures may not accurately represent the conditions from the TSP image. The aft-most shock location (seen in Frame A data in Figure 31) occurs at an approximate $\mathrm{x} / \mathrm{c}$ of 0.50 , which could explain why the TSP image showed transition in this region. The variation in pressure distribution character may be due to small variations in Mach or twist deflection during data acquisition. This phenomenon was observed at all four angles of attack primarily on the outboard region of the wing, most commonly between Rows E and I. The inconsistency between chordwise transition location and pressure distributions makes determining the dominant transition mechanism or estimating critical $\mathrm{N}$-factor especially challenging.

One of the test objectives for the experiment was to characterize the NTF laminar flow testing capabilities, including determining critical $\mathrm{N}$-factors for the facility. The general procedure for determining the critical $\mathrm{N}$-factor involves both computational stability analysis and experimental transition locations. Two previous examples, Figures 23 and 24, show the N-factor growth with the transition location marked on the plot. From Figure 23, it can be deduced that the TS critical N-factor would be approximately 5.5 at that condition, as that is the $\mathrm{N}$-factor at the location of transition in the tunnel. Similarly, Figure 24 implies the midchord CF critical N-factor is greater than 5.9 for the condition (greater than 5.9 because it is wedges coalescing rather than a natural transition front). Measuring several spanwise locations at multiple conditions can provide a sense of variation in the critical $\mathrm{N}$-factor levels for each mechanism. Due to the limited visibility of natural transition fronts due to bypass transition at higher Reynolds numbers, the data acquired at 17.5 million Reynolds number and greater cannot be confidently used in determining dominant transition mechanisms or critical N-factors. Additionally, the outboard regions of the wing often did not have stability analysis results at the transition location, which limits the understanding of transition mechanisms in those regions. However, the data available still provide insight into the CRM-NLF transition mechanisms and NTF laminar flow environment. It was determined that the angle of attack of 1.5 degrees was likely transitioning due to midchord CF growth outboard of Row B at all Reynolds numbers between 10.0 and 15.0 million. The angles of attack between 2.0 and 3.0 degrees showed TS growth to be the dominant mechanism of transition across the span of the wing at the 15.0 million condition. A scatter plot of $\mathrm{N}$-factor values for both CF and TS is shown in Figure 32. The data in this graph are the maximum $\mathrm{N}$-factor values reached forward of the experimental transition locations across all angles of attack and Reynolds numbers studied thus far in this paper. Many of these values are artificially low due to stability analysis calculations terminating prior to the transition location, as well as unrealistically forward experimental transition locations due to bypass or leading-edge transition. Figure 32 provides an idea of the spread of N-factors, but does not include any information on which of these $\mathrm{N}$-factors are actually critical at a given condition. The 15 million Reynolds number conditions can be used to provide an estimate of the range of critical $\mathrm{N}$-factors seen on the CRM-NLF in the NTF. Figure 33 shows only the N-factors that were deemed critical (i.e., the N-factor of the dominant transition mechanism at the experimental transition location) from the angle-of-attack sweep. The open symbols seen outboard are representative of the stations where the computational stability analysis results did not reach the experimental chordwise transition location, which means the critical $\mathrm{N}$-factors in this region are artificially low. The squares are TS dominant transition and triangles are $\mathrm{CF}$ dominant transition. This chart shows the critical $\mathrm{N}$-factors typically fall within $4-7$ for both CF and TS, and would likely be higher for the open symbol conditions. This range of critical Nfactors is consistent with findings published from a laminar flow test previously conducted in the NTF [20]. 


\section{Summary of Findings from the CRM-NLF Wind Tunnel Test}

In the Introduction section of this paper, the three objectives for the wind tunnel test were stated. In spite of some of the challenges of laminar flow wind tunnel testing described in the previous sections, progress was made to address each of these goals. The first goal was to validate the CATNLF design method and analysis tools, and several pieces of evidence that supported its validation were acquired in the NTF. A key confirmation was that, for multiple conditions tested, the transition Reynolds number exceeded the historic boundary for NLF at comparable leading-edge sweeps. This boundary is shown in Figure 34 along with the maximum transition Reynolds number observed on the CRM-NLF in the NTF, which nearly doubles the value that was previously seen at comparable sweeps in a flight or wind tunnel NLF experiment [27]. Additionally, the laminar flow extended past the leading-edge region over parts of the span at all Reynolds numbers tested (10 - 30 million), suggesting the CF suppression approach using CATNLF pressure architecture is valid. However, the increase in bypass wedges and attachment line transition at higher Reynolds numbers limited the ability to fully assess this aspect. At moderate Reynolds numbers (15 million and below), the pretest computational predictions and experiment transition fronts matched fairly well, an example of which is shown in Figure 35. This agreement suggests that the tools, design philosophy, and assumptions used in the CATNLF design process were reasonable. Finally, the leading-edge experimental pressure data provide confirmation that the pressure architecture used to attenuate the CF growth (rapid acceleration at the leading edge followed by a nearly flat pressure gradient) was achieved on the wind tunnel model. The CRM-NLF geometry and some wind tunnel data will be released to the public on the CRM website at https://commonresearchmodel.larc.nasa.gov/crm-nlf/.

The second test objective was to characterize the NTF for laminar flow testing, with a specific target of refining the range of critical $\mathrm{N}$-factor values previously determined. Thus far, the results from this test have not significantly narrowed the previously estimated range of $4-8$ for TS transition [20], but the present analysis does suggest a range of 4- 7 for both TS and CF (CF critical $\mathrm{N}$-factor had not previously been experimentally determined). These estimates are based on using newly computed post-test flow solutions, which matched the experimental pressures fairly well. Work is underway to develop a consistent way of infilling the experimental pressures to provide sufficient detail for use in stability analysis calculations. Performing stability analysis directly on the experimental pressures is expected to improve the critical N-factor estimates. Additionally, a method to better correlate the TSP images to the frame data of experimental pressures could eliminate the forward termination of stability analysis, and is also expected to improve the critical $\mathrm{N}$-factor estimates. As with the first goal, the turbulent wedges and occurrence of bypass attachment line transition at the higher Reynolds numbers limited the ability to more fully investigate the second objective of characterizing the NTF critical N-factors.

The final test objective was to establish best practices for laminar flow testing, specifically in a cryogenic facility and is, by definition, an on-going process. The most significant new experimental procedure from the CRM-NLF test was the successful implementation of the carbon-based heating layer with the TSP for laminar flow visualization. After considerable refinement before and during the test, the final version of the heating layer provided TSP images with the same or better detail than the $\mathrm{LN}_{2}$ injection approach, and improved the quality and efficiency of data acquisition. One ongoing challenge with laminar flow wind tunnel testing that still needs addressing is the occurrence of bypass transition. The primary limitation on the data analysis was due to the number of turbulent wedges that occur when testing at high Reynolds numbers, as well as wedges that accumulate during testing. The CATNLF design method necessitated painting the leading edge of the wing with TSP to visualize transition due to CF peaks in that region. The initial paint surface prior to testing was highly-polished, with an average surface roughness measured as approximately $1.3 \mu \mathrm{in}$. Frequent sanding and polishing occurred during testing such that the average surface roughness measured approximately $1.0 \mu \mathrm{in}$ after testing. However, it is likely that the paint had local imperfections that could not be removed with the sanding and polishing procedure, such as divots or scratches. For the CRM-NLF test, the best operational procedure to reduce bypass transition was this periodic sanding and polishing to remove surface imperfections, however, this procedure reduced testing efficiency and cost time and resources to reestablish the test conditions in the cryogenic tunnel. Two areas of study that have come from the efforts to eliminate or reduce bypass transition during laminar flow wind tunnel testing include: 1) a filler material that could address imperfections when sanding is less effective, such as divots or deeper scratches, and 2) a surface coating that would harden the paint to prevent or reduce the surface damage from particle impact during testing. Both would need to provide the required surface finish suitable for laminar flow and work in a cryogenic test environment, without adversely impacting the TSP luminosity.

In view of the promising, but limited, confirmation of the CATNLF design method, a series of flight tests have been proposed to more thoroughly evaluate the method and advance the technology. While the design Reynolds number of 30 million was achieved in the NTF, the TSP images at that condition had very limited visibility. This was primarily due to the low critical $\mathrm{N}$-factors and excess bypass transition, which are factors of the tunnel environment and 
limitations on model size. The flight environment should provide a higher critical $\mathrm{N}$-factor level, with previous estimates around 10. In addition, the scale of the proposed flight test article is about 5 times greater than the CRMNLF wing, which produces a thicker boundary layer, making it less sensitive to bypass transition due to particles.

A series of three flight tests are currently being developed, all utilizing an F-15 aircraft testbed at the NASA Armstrong Flight Research Center. Each flight test will have a test article mounted beneath the F-15 on the Centerline Instrument Pylon (CLIP). The first flight test in the series is scheduled for 2019, and is a flight evaluation of the carbon-based heating layer technology, similar to what was used for the CRM-NLF test in the NTF. An existing laminar flow test article will be painted with the carbon-based heating layer for use with infrared technology to visualize laminar flow in flight. As in the wind tunnel, the use of this heating layer should increase the efficiency of data acquisition and provide more consistent test conditions. The second flight test will involve a new rake that has been designed and instrumented for the purpose of surveying the flow field under the F-15 where test articles are mounted. This flight test, scheduled for 2020, will quantify several parameters relevant for laminar flow testing, and provide valuable insight into results from previous supersonic NLF flight tests [28], as well as this CATNLF flight test series. The final flight test will be to validate the CATNLF method in a flight relevant environment. This test will involve a new wing test article designed using the CATNLF method and is currently scheduled for the end of 2020. The wing will have a leading-edge sweep of 35 degrees, a reference chord of 5.9 feet, and a span of 3.4 feet. The design Mach number is 0.85 and the maximum test Reynolds number will be 31 million, based on the reference chord. Altitude will be varied and infrared technology will be used to observe laminar flow extents on the CATNLF flight test article at a variety of Reynolds numbers. The same CATNLF flight test article is also slated to be tested in a wind tunnel environment, likely the NTF, to enable a direct comparison of laminar flow extents in flight and wind tunnel environments. The success of the CRM-NLF in the NTF has led to this series of flight tests, which will aid in advancing the CATNLF technology.

\section{Concluding Remarks}

A new NLF design method, CATNLF, was experimentally investigated in a wind tunnel test in the NTF at the NASA Langley Research Center in 2018. The CATNLF design method alters the shape of the wing airfoils to obtain specific pressure distribution characteristics that are known to control boundary layer instabilities. The novel aspect of the CATNLF design method is the crossflow attenuation on components with high sweep and Reynolds numbers, such as the main wings of a typical transonic transport. If successful, the CATNLF method enables the known laminar flow performance benefit without incurring historic crossflow penalties, such as the slower cruise speeds when unsweeping the wing or the added weight, complexity, and cost when employing a suction system. The CATNLF method was used to design a wind tunnel model, referred to as the CRM-NLF. A semispan model of the CRM-NLF was tested at transonic cruise speeds at flight relevant Reynolds numbers in the NTF. The test aimed to address three primary test objectives: validate the CATNLF design method and analysis tools, characterize the NTF laminar flow testing capabilities, and establish best practices for laminar flow wind tunnel testing. This paper provided experimental data and computational results to evaluate the CRM-NLF laminar flow characteristics, as well as assess the three test objectives.

Significant extents of laminar flow were observed on the CRM-NLF in the NTF at several test conditions. The data were primarily acquired at transonic speeds, near-cruise angles of attack, and Reynolds numbers based on mean aerodynamic chord between 10 and 30 million. This paper explored the effects of angle of attack and Reynolds number on laminar flow extents. Computational flow solver and stability analysis results were used to determine the dominant mechanism of transition at a variety of conditions. The computational data were also used to determine the critical Nfactors for both TS and CF in the NTF. Several challenges were encountered while performing this data analysis relating to the increased bypass transition that occurs at high Reynolds numbers in a wind tunnel environment, as well as dynamic pressure readings on the wing. Even with the limitations of the higher Reynolds number cases, the three test objectives were addressed to some extent in this paper.

The CRM-NLF saw extents of laminar flow, based on transition Reynolds number, that nearly doubled those seen in previous flight and wind tunnel NLF experiments at comparable leading-edge sweep angles. Additionally, the computational pretest predictions and experimental transition fronts showed close agreement. These two key results from the test suggest the CATNLF design method was successful at attenuating the crossflow growth on the wing. This initial confirmation has led to future work that includes a series of flight tests to advance the CATNLF technology in a flight relevant environment. 


\section{Acknowledgments}

This research is funded by the NASA Advanced Air Transport Technology Project within the Advanced Air Vehicles Program. Resources supporting some computational results in this paper were provided by the NASA HighEnd Computing (HEC) Program through the NASA Advanced Supercomputing (NAS) Division. The authors would like to acknowledge several key researchers who were critical to the success of the CRM-NLF wind tunnel test, including Melissa Rivers, David Chan, Dr. Neal Watkins, and Scott Goodliff. The authors also would like to thank Kyle Goodman and Dr. Sarah Peak, as well as the many instrumentation technicians at the National Transonic Facility, for their invaluable contribution to the acquisition of the experimental data. Additionally, the authors thank Lewis Owens for offering his continued support and expertise in the computational and experimental investigation of boundary layer transition. Finally, the authors thank Donald Saxer, Lonnie Brown, Dr. Richard Wahls, Scott Anders, and Susan Wilz for supporting the planning and execution of the wind tunnel test.

\section{References}

[1] Norris, G., “757 EcoDemo Focuses On Laminar and Active Flow”, Aviation Week \& Space Technology, March $25,2015$. https://awin.aviationweek.com/ArticlesStory/tabid/975/Status/IPAddress/id/341632bd-4510-439f-bcd6d236474194d3/Default.aspx

[2] Norris, G., "Picture: Boeing plans plain grey natural laminar flow nacelles for 787s in bid to reduce fuel burn", Flight International, 11 July, 2006. https://www.flightglobal.com/news/articles/picture-boeing-plans-plain-grey-natural-laminarflow-nacelles-for-787s-in-bid-to-reduce-fuel-207769/

[3] Fujino, M., "Design and Development of the HondaJet", AIAA International Air and Space Symposium and Exposition: The Next 100 Years, AIAA 2003-2530, July 2003.

[4] Warwick, G., "Europe Begins Laminar-Flow Flight Tests For Future Airline", Aviation Week \& Space Technology, October 5, 2017. https://awin.aviationweek.com/ArticlesStory.aspx?id=598c12f2-e409-4d55-b6ba-a4ab1b3bf41a

[5] Geyr, H., "DLR A320 Advanced Technology Research Aircraft prepared for AFLoNext flight test campaign”, 2019. https://event.dlr.de/en/ila2018/aflonext/

[6] Saric, William S.; Carrillo, Ruben, B., Jr.; and Reibert, Mark S.: Leading-Edge Roughness as a Transition Control Mechanism. AIAA 1998-0781, January 1998.

[7] Lynde, M. N. and Campbell, R. L., "Expanding the Natural Laminar Flow Boundary for Supersonic Transports", 34th AIAA Applied Aerodynamic Conference, AIAA AVIATION Forum, AIAA 2016-4327, June 2016.

[8] Campbell, R. L., and Lynde, M. N., "Natural Laminar Flow Design for Wings with Moderate Sweep", 34th AIAA Applied Aerodynamic Conference, AIAA AVIATION Forum, AIAA 2016-4326, June 2016.

[9] Lynde, M. N., and Campbell, R. L., "Computational Design and Analysis of a Transonic Natural Laminar Flow Wing for a Wind Tunnel Model," AIAA-2017-3058, June 2017.

[10] Lynde, M. N., Campbell, R. L., Rivers, M. B., Viken, S. A., Chan, D. T., Watkins, A. N., and Goodliff, S. L., "Preliminary Results from an Experimental Assessment of a Natural Laminar Flow Design Method", AIAA 2019-2298, January 2019.

[11] Campbell, R. L., "Efficient Viscous Design of Realistic Aircraft Configurations," AIAA-98-2539, June 1998.

[12] Frink, N.T., Pirzadeh, S.Z., Parikh, P.C., Pandya, M.J., and Bhat, M.K., "The NASA Tetrahedral Unstructured Software System," The Aeronautical Journal, Vol. 104, No. 1040, October 2000, pp.491-499.

[13] Chang, C.-L., "The Langley Stability and Transition Analysis Code (LASTRAC): LST, Linear and Nonlinear PSE for 2-D, Axisymmetric, and Infinite Swept Wing Boundary Layers," AIAA 2003-0974, 2003.

[14] Wie, Y.-S., "BLSTA: A Boundary Layer Code for Stability Analysis," NASA CR 4481, 1992.

[15] Vassberg, J.C. and Rivers, S.M., "Development of a Common Research Model for Applied CFD Validation", AIAA 20086919, August 2008.

[16] Poll, D. I. A., "Some Observations of the Transition Process on the Windward Face of a Long Yawed Cylinder," J. Fluid Mech., Vol. 150, 1985, pp. 329-356.

[17] National Transonic Facility (NTF), "NTF User Guide", NASA Document SP-2011-5-110-LaRC, NASA Langley Research Center, February 2012.

[18] Wahls, R.A., "The National Transonic Facility: A Research Retrospective (Invited)”, AIAA 2001-0754, 39th AIAA Aerospace Sciences Meeting and Exhibit, January 2001.

[19] King, R. A., Andino, M. Y., Melton, L., Eppink, J., and Kegerise, M. A., "Flow Disturbance Measurements in the National Transonic Facility", AIAA Journal, Vol. 52, No. 1, January 2014, pp. 116-130.

[20] Crouch, J., Sutanto, M., Witkowski, D., Watkins, A., Rivers, M., and Campbell, R., "Assessment of the National Transonic Facility for Natural Laminar Flow Testing", 48th AIAA Aerospace Sciences Meeting Including the New Horizons Forum and Aerospace Exposition, Aerospace Sciences Meetings, AIAA 2010-1302, January 2010.

[21] Rivers, M., Lynde, M., Campbell, R., Viken, S., Chan, D., Watkins, A., and Goodliff, S., "Experimental Investigation of the NASA Common Research Model with a Natural Laminar Flow Wing in the NASA Langley National Transonic Facility", AIAA 2019-2189, January 2019.

[22] Iyer, V., and Everhart, J., "Application of Pressure-Based Wall Correction Methods to Two NASA Langley Wind Tunnels", AIAA 2001-2472, June 2001.

[23] Hammer, M., Popernack, T., Owens, L., and Wahls, R., "Using temperature sensitive paint technology", 40th AIAA 
Aerospace Sciences Meeting \& Exhibit, Aerospace Sciences Meetings, AIAA 2002-0742, 2002.

[24] Klein, C. and Henne, U., "Combination of Temperature Sensitive Paint and Carbon Nanotubes for Transition Detection", 53rd AIAA Aerospace Sciences Meeting, AIAA SciTech, AIAA 2015-1558, January 2015.

[25] Goodman, K.Z., Lipford, W.E., and Watkins, A.N., "Boundary-Layer Detection at Cryogenic Conditions Using Temperature Sensitive Paint Coupled with a Carbon Nanotube Heating Layer", Sensors 2016, 16, 2062.

[26] Watkins, A.N., Goodman, K.Z., and Peak, S.M., "Transition Detection at Cryogenic Temperatures Using a Carbon-Based Resistive Heating Layer Coupled with Temperature Sensitive Paint”, 2019 AIAA SciTech Forum, AIAA 2019-2191, January 2019.

[27] Malik, M.R., Crouch, J.D., Saric, W.S., Lin, J.C., and Whalen, E.A., “Application of Drag Reduction Techniques to Transport Aircraft", Encyclopedia of Aerospace Engineering, 2015.

[28] Owens, L., Beeler, G., King, R., Chou, A., Balakumar, P., and Banks, D., "Supersonic Crossflow Transition Control in Ground and Flight Tests", AIAA 2019-1651, January 2019. 


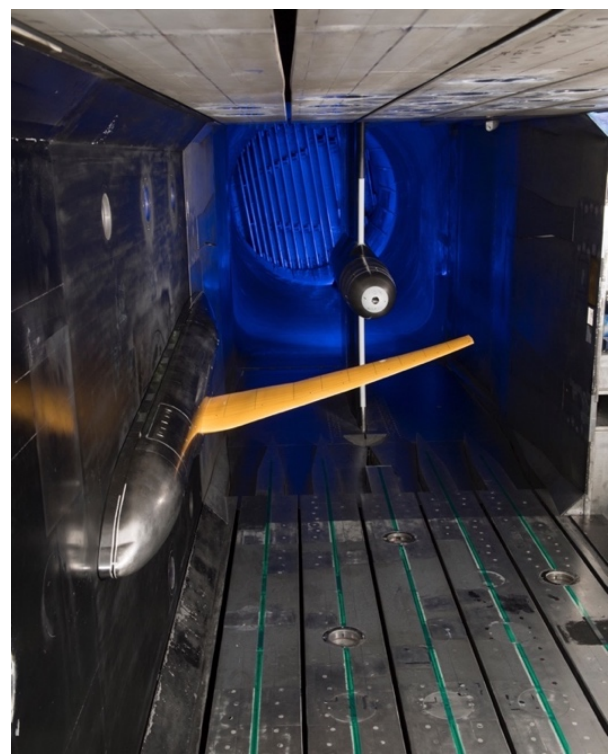

Figure 1. CRM-NLF semispan model mounted to the sidewall of the NTF.

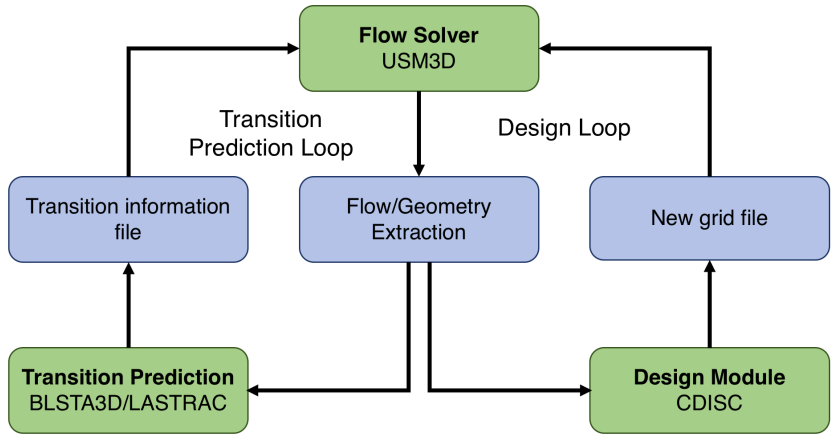

Figure 2. Flow chart of the CDISC design and analysis process.

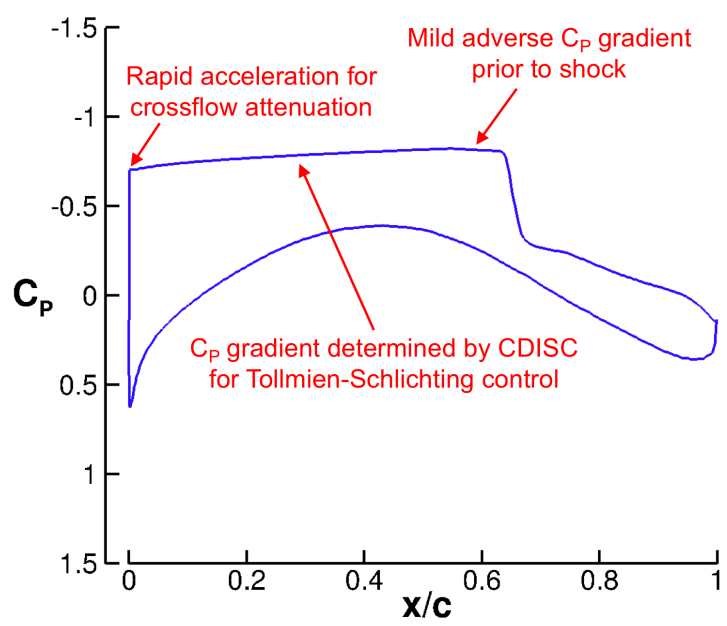

Figure 3. Example CATNLF pressure distribution for a transonic transport. Key features of the pressure distributions are identified. 


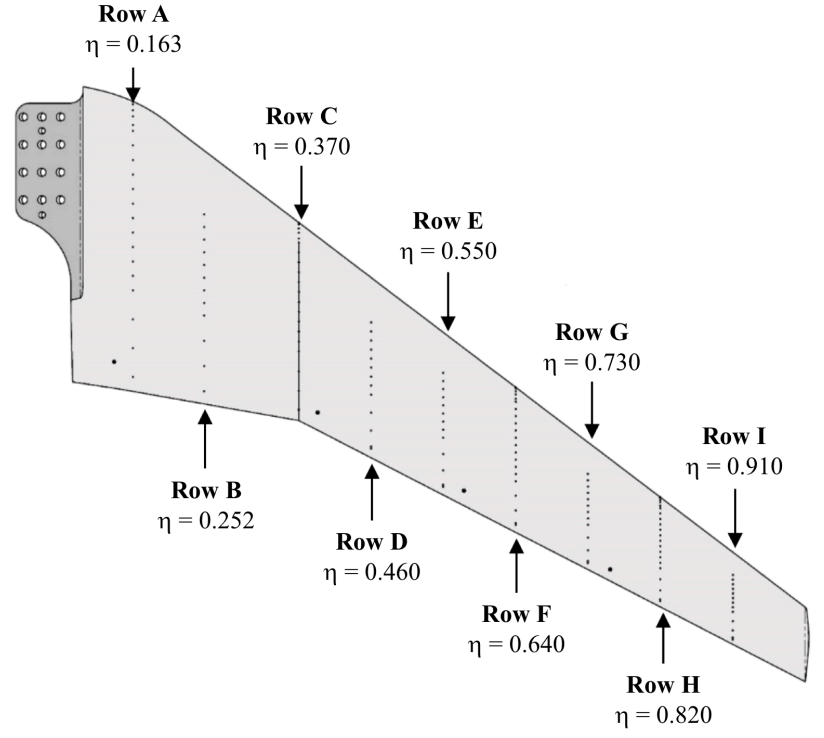

Figure 4. Planform view of the CRM-NLF wing showing pressure orifice rows on the upper surface.

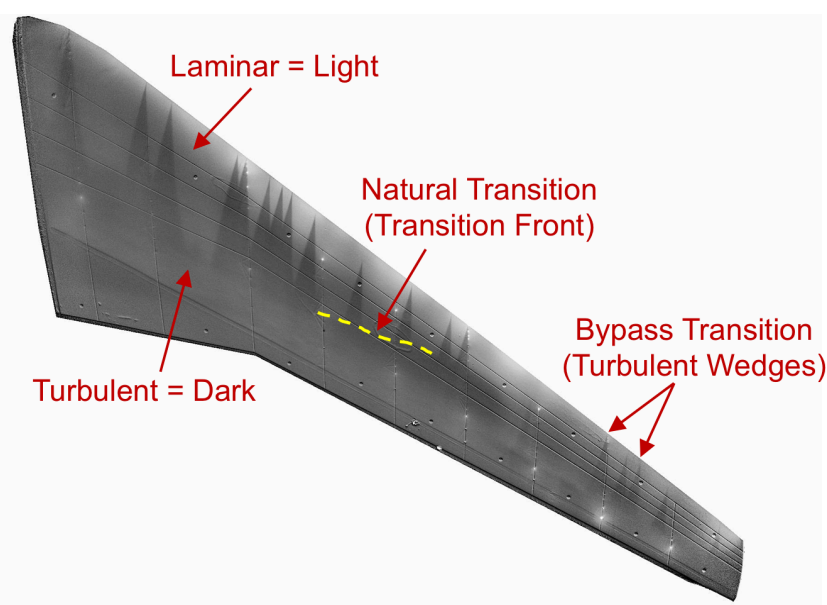

Figure 5. Example TSP image with key features noted. 


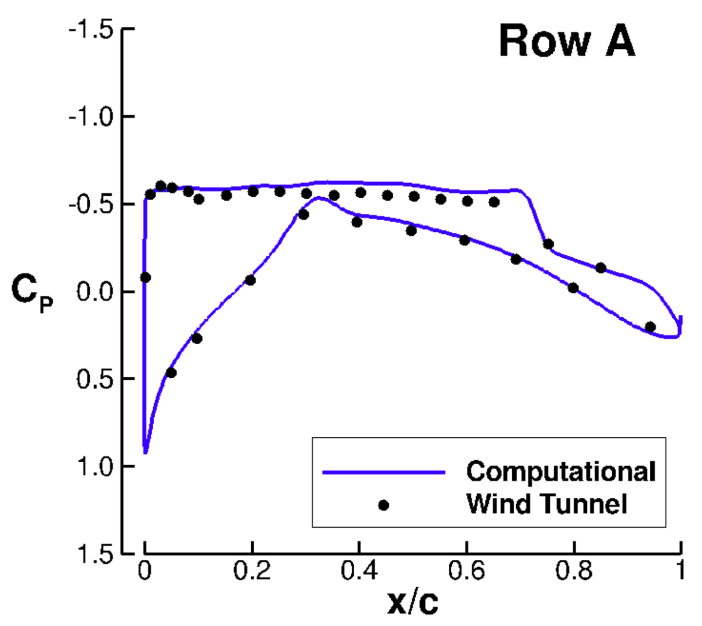

a) $\operatorname{Row} A(\eta=0.163)$

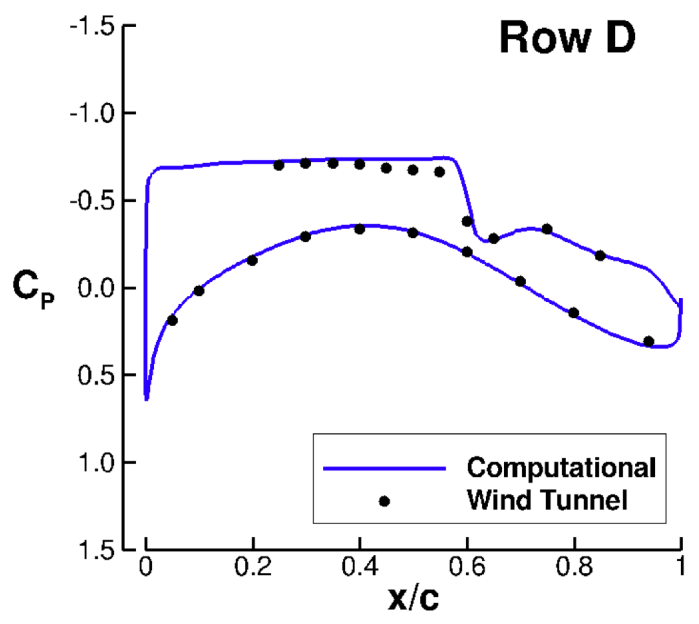

c) $\operatorname{Row} D(\eta=0.460)$

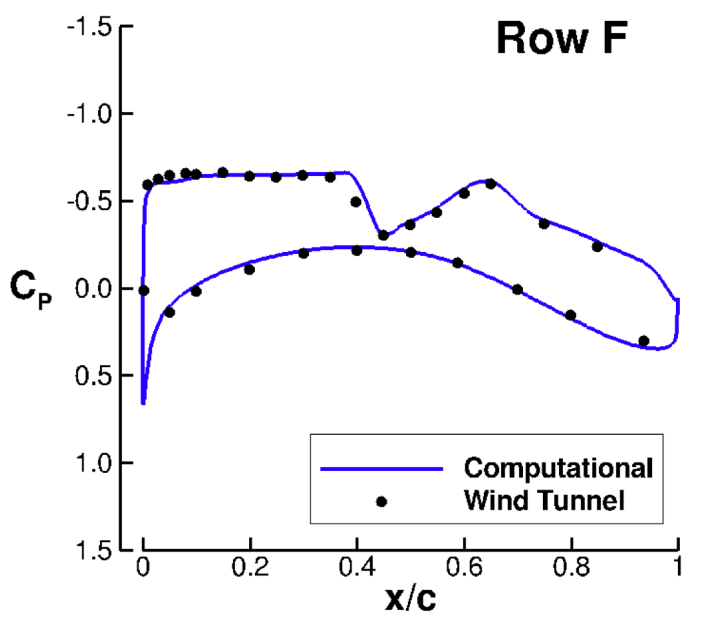

e) $\operatorname{Row} F(\eta=0.640)$

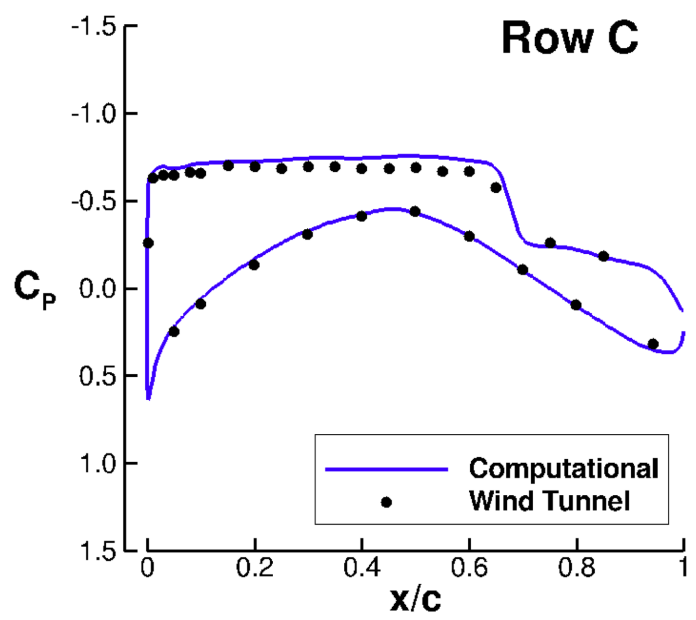

b) $\operatorname{Row} C(\eta=0.370)$

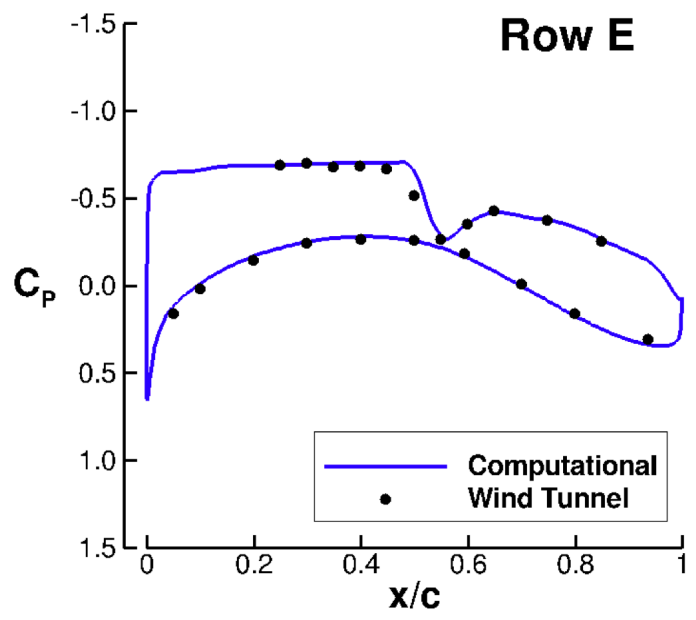

d) $\operatorname{Row} E(\eta=0.550)$

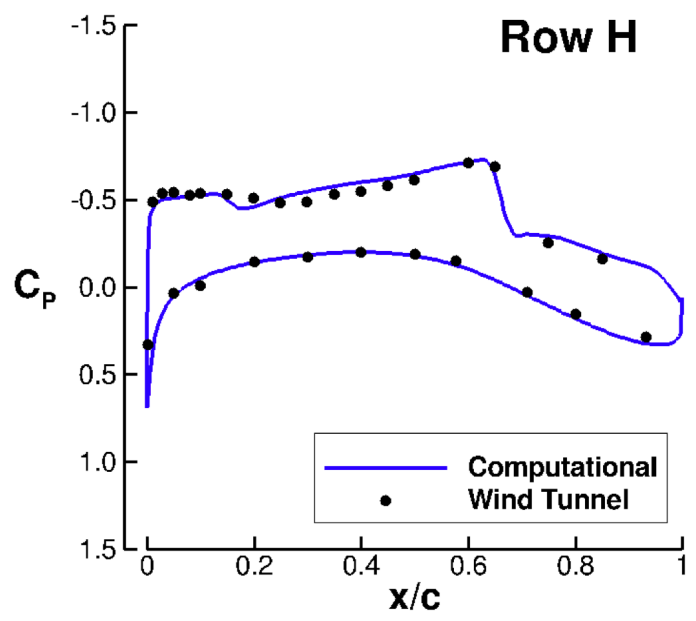

f) $\operatorname{Row} H(\eta=0.820)$

Figure 6. Pressure distributions across the span comparing computational results and wind tunnel pressure data. All data are from $M=0.86, \alpha=2.0 \mathrm{deg} . \mathrm{T}_{\mathrm{T}}=40^{\circ} \mathrm{F}$, and $\operatorname{Re}_{\mathrm{MAC}}=15$ million. 


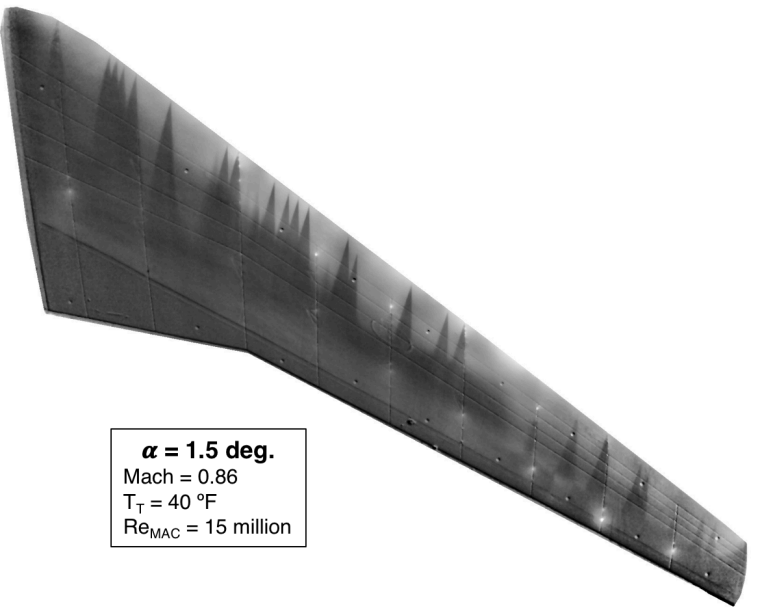

a) $\alpha=1.5 \mathrm{deg}$.

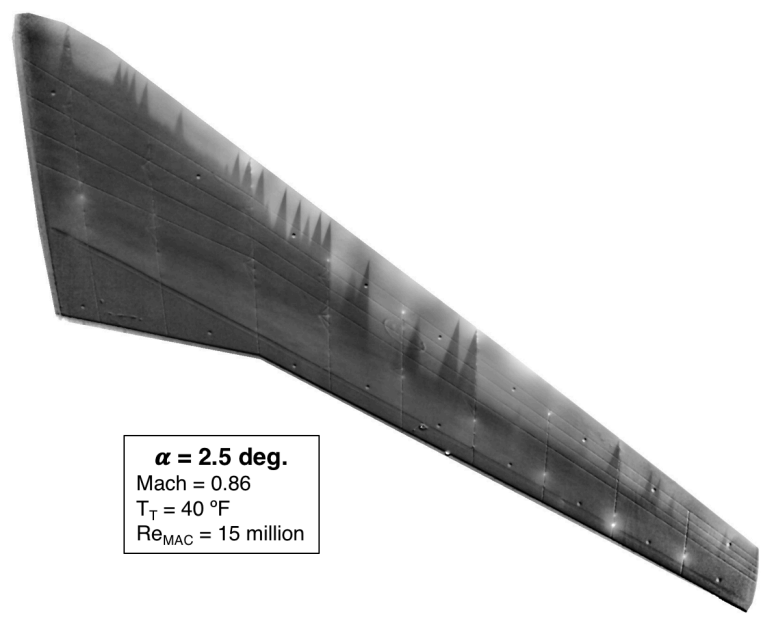

c) $\alpha=2.5 \mathrm{deg}$.

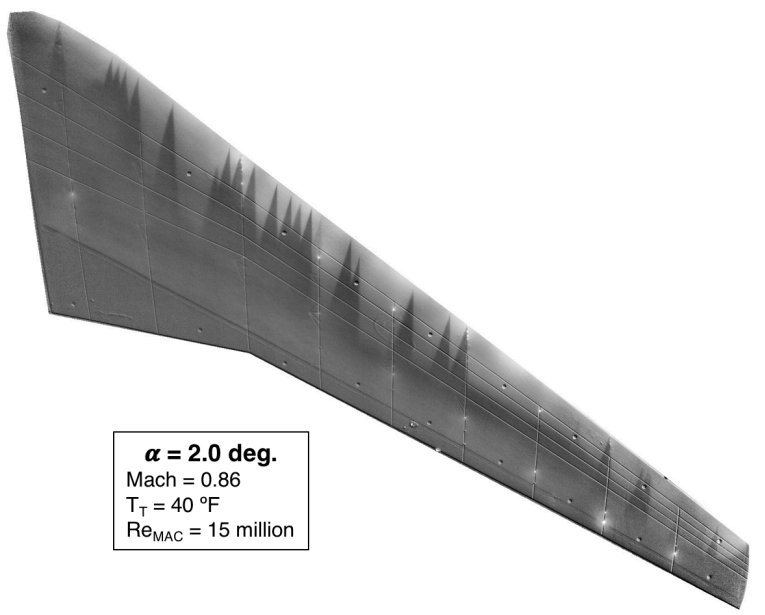

b) $\alpha=2.0 \mathrm{deg}$.

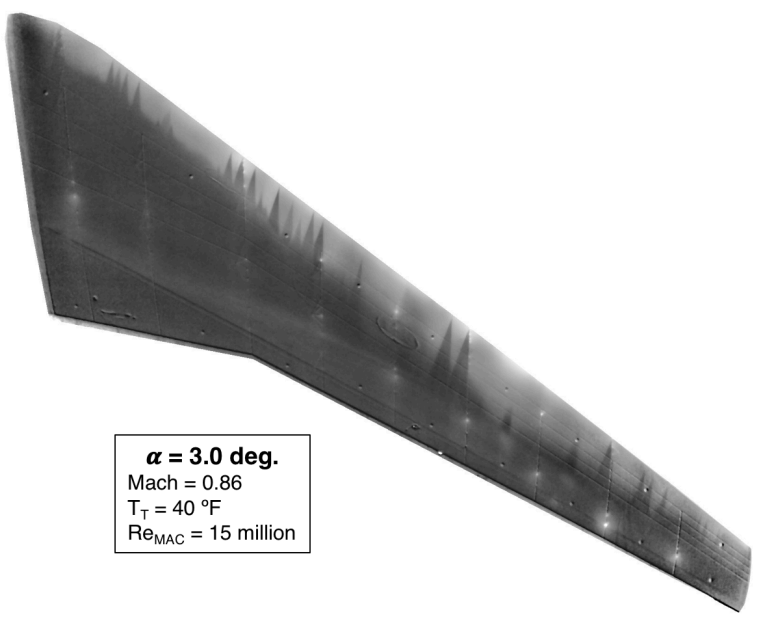

d) $\alpha=3.0 \mathrm{deg}$.

Figure 7. TSP images from an angle-of-attack sweep at $M=0.86, T_{T}=40{ }^{\circ} \mathrm{F}$, and $\operatorname{Re}_{\mathrm{MAC}}=15$ million.

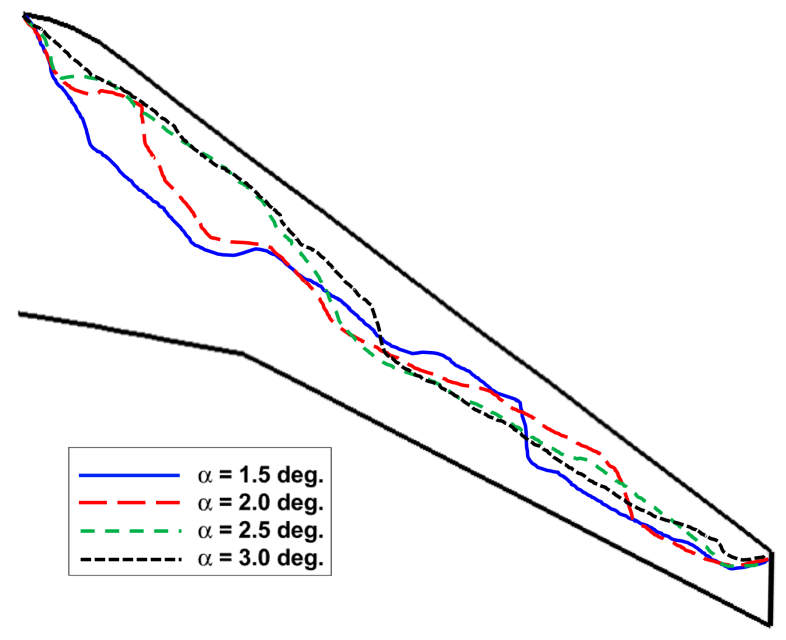

Figure 8. Experimental transition fronts from the angle-of-attack sweep corresponding to the TSP images in Figure 7 at $M=0.86, T_{T}=40^{\circ} \mathrm{F}$, and $R_{M A C}=15$ million. 


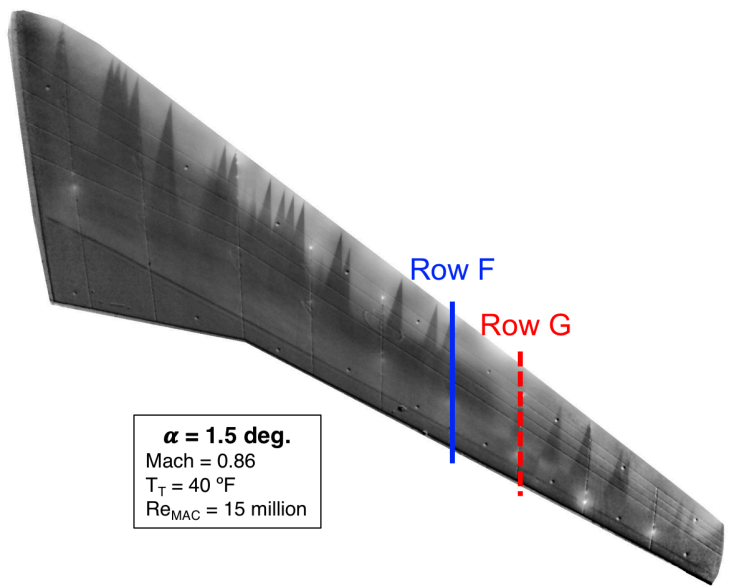

a) TSP image

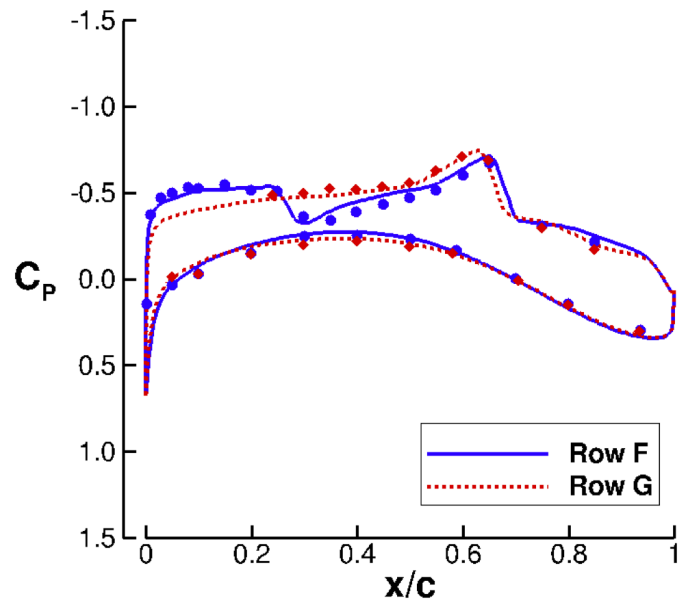

b) Computational (lines) and experimental (symbol) pressure distributions

Figure 9. TSP image and pressure distributions showing sudden aft shift in transition location between Rows $F(\eta=0.640)$ and $G(\eta=0.730)$. All data from $M=0.86, \alpha=1.5$ deg., $T_{T}=40^{\circ} \mathrm{F}$, and $\operatorname{Re}_{M A C}=15$ million.

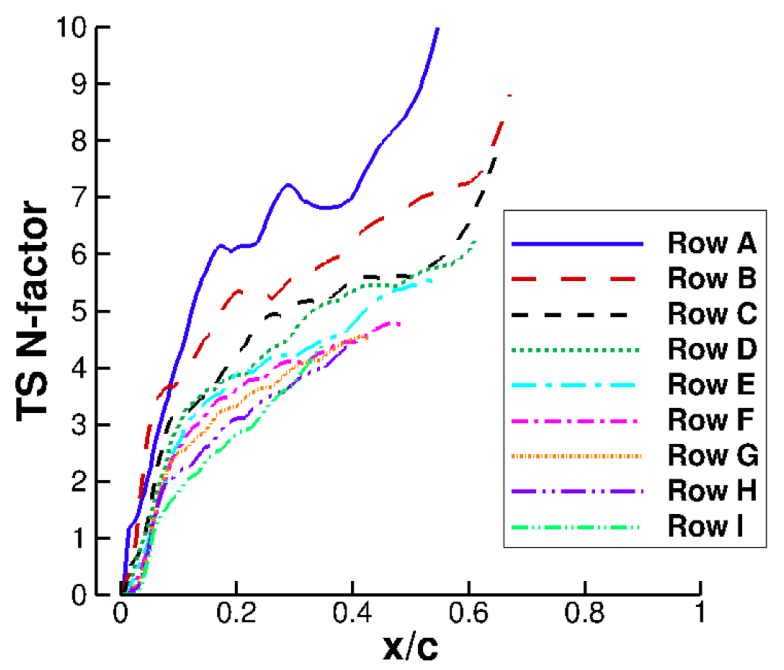

a) TS

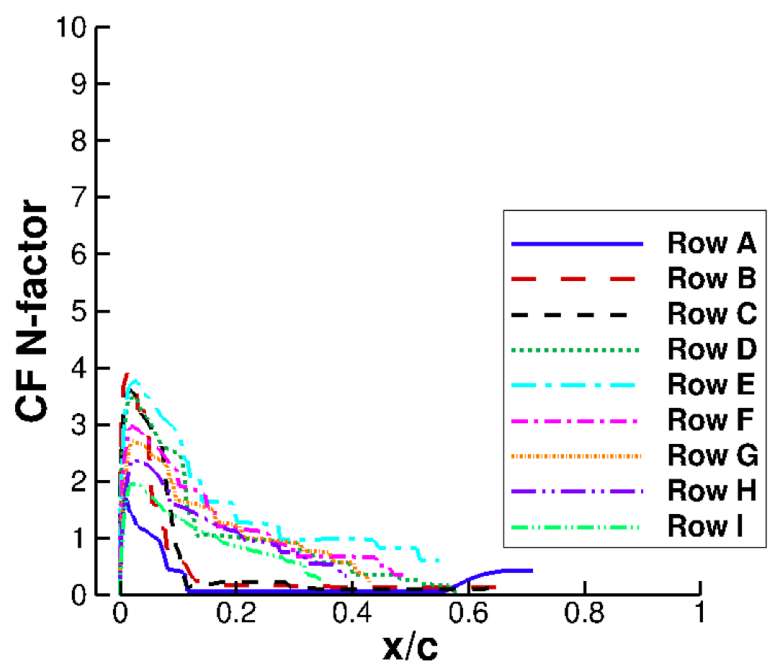

b) $\mathbf{C F}$

Figure 10. Computational N-factor growth for TS and CF versus $x / c$ across the span of the CRM-NLF wing. Data corresponds to $M=0.86, \alpha=2.5 \mathrm{deg}$., $T_{T}=40^{\circ} \mathrm{F}$, and $\operatorname{Re}_{\mathrm{MAC}}=15$ million. 


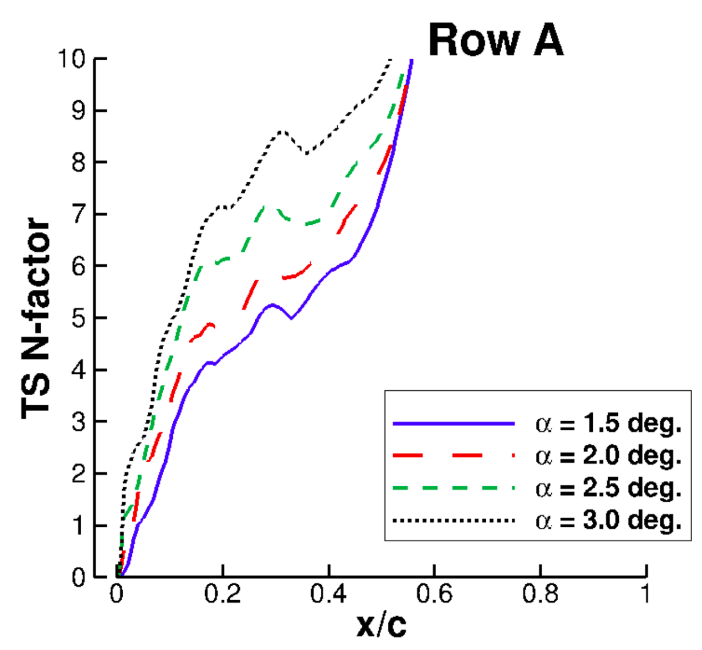

a) TS growth at $\operatorname{Row} A(\eta=0.163)$

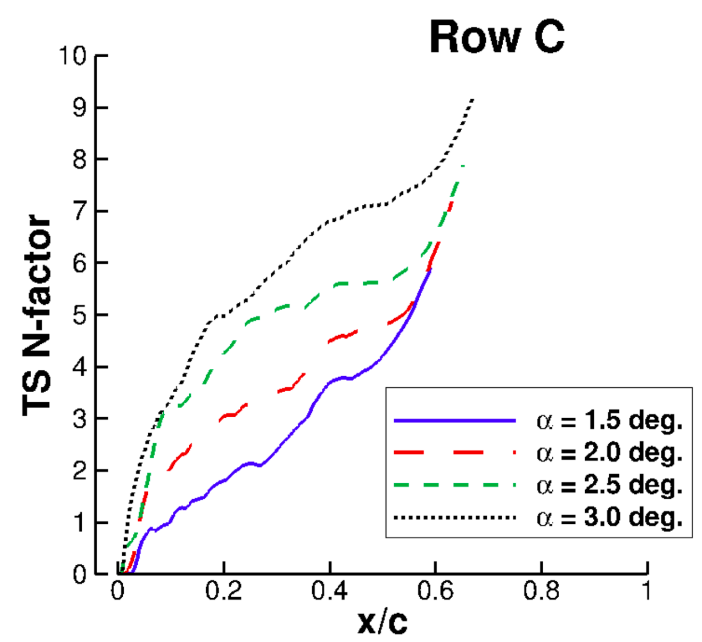

c) TS growth at $\operatorname{Row} C(\eta=0.370)$

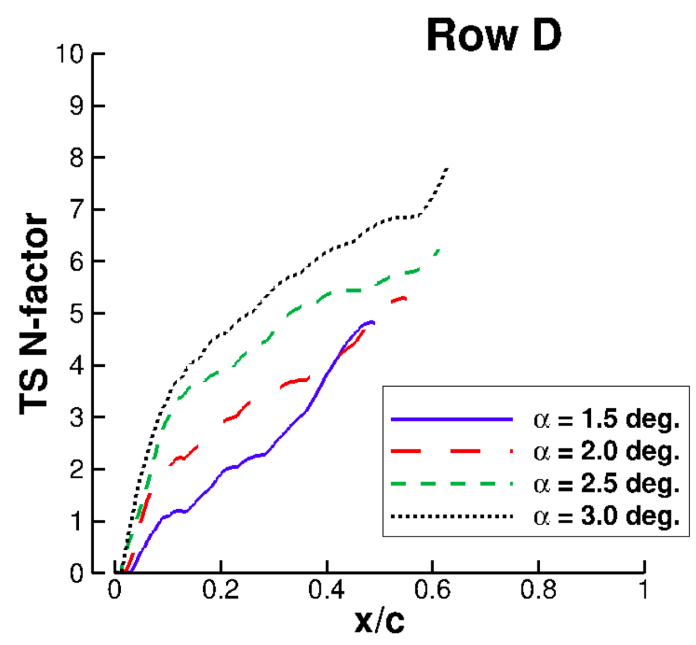

e) TS growth at $\operatorname{Row} D(\eta=0.460)$

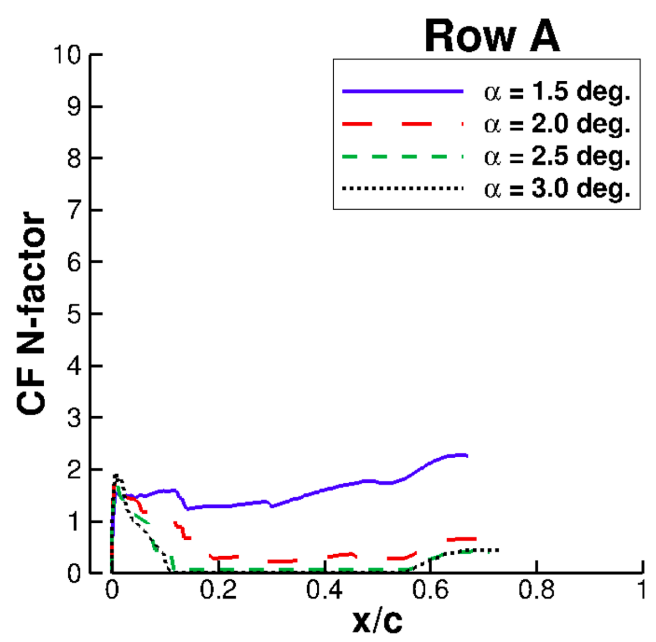

b) CF growth at $\operatorname{Row} A(\eta=0.163)$

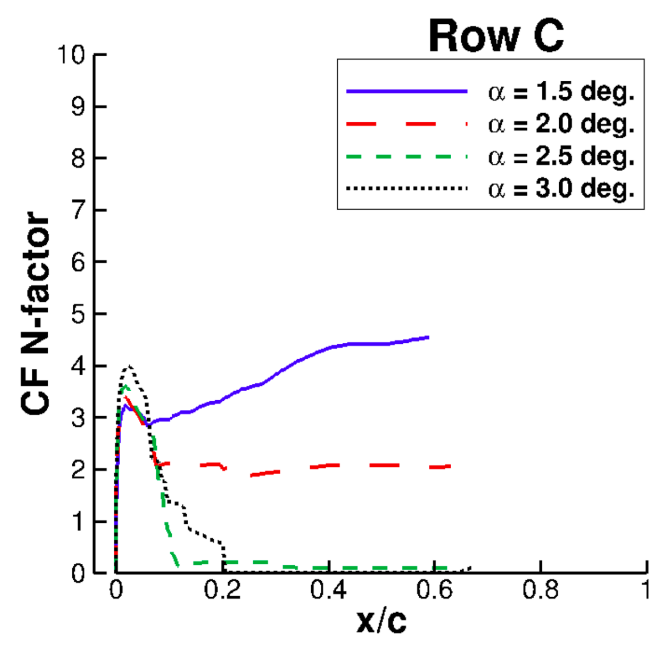

d) $C F$ growth at $\operatorname{Row} C(\eta=0.370)$

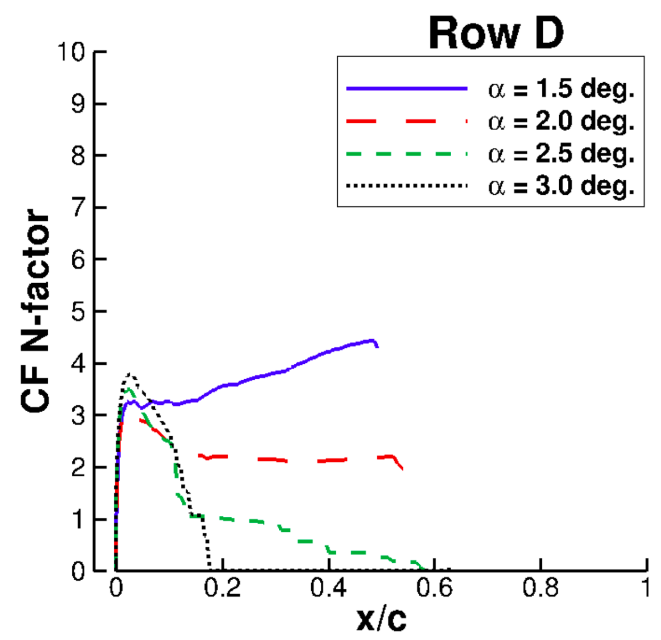

f) CF growth at $\operatorname{Row} D(\eta=0.460)$

Figure 11. TS and CF N-factor growth versus $x / c$ at several spanwise stations for the angle-of-attack sweep from $M=$ $0.86, T_{T}=40{ }^{\circ} \mathrm{F}$, and $\operatorname{Re}_{\mathrm{MAC}}=15$ million. 


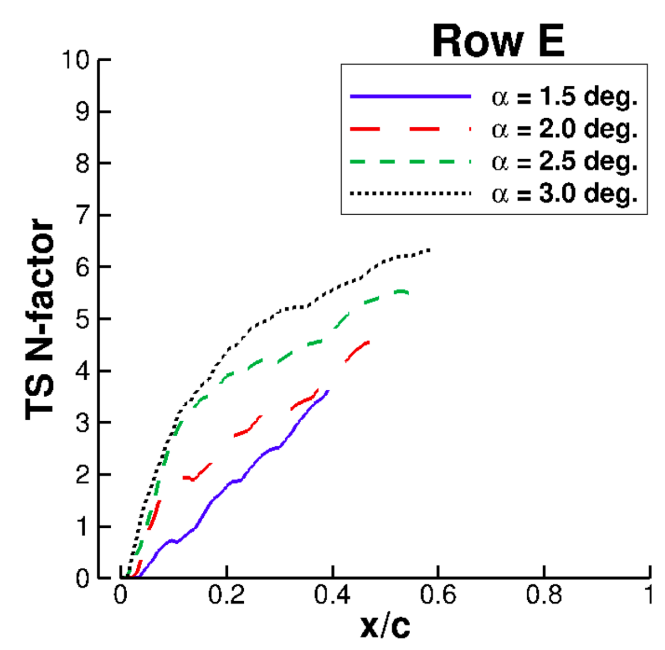

g) TS growth at Row $E(\eta=0.550)$

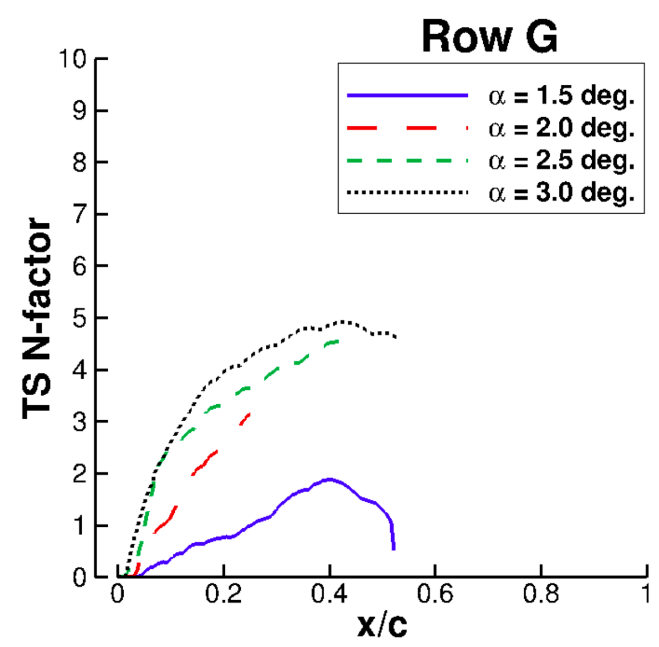

i) TS growth at $\operatorname{Row} G(\eta=0.730)$

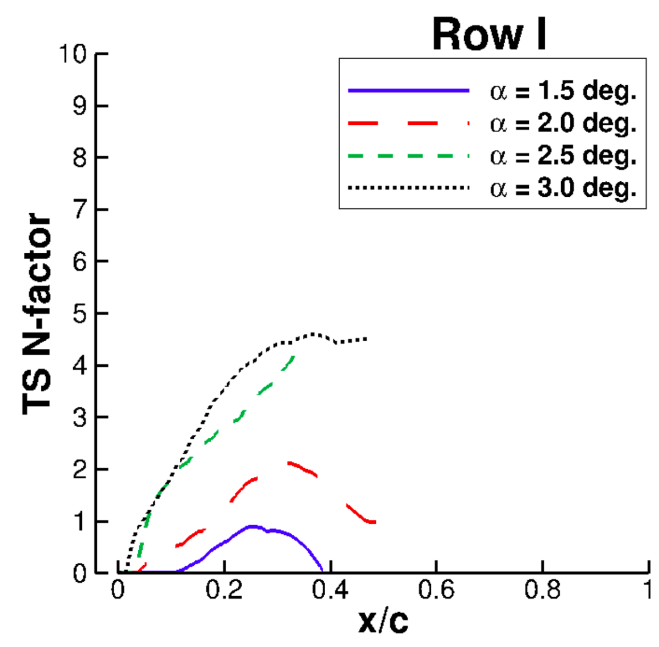

k) TS growth at Row $I(\eta=0.910)$

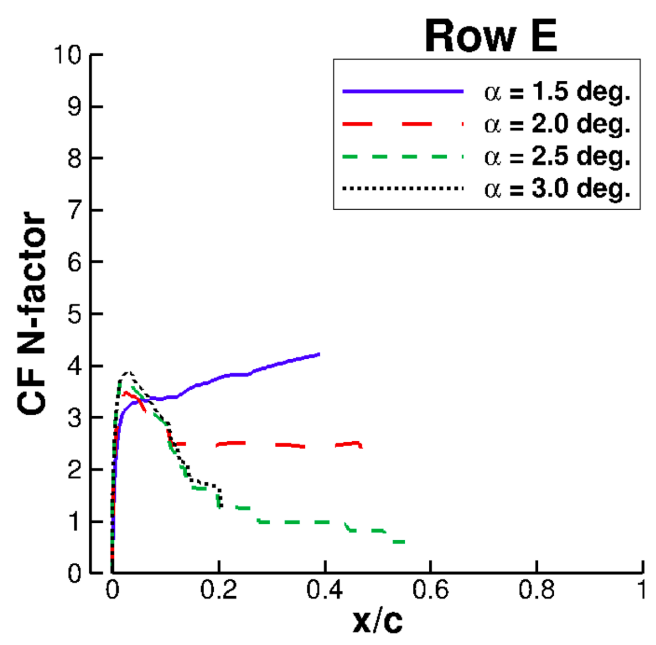

h) CF growth at Row $E(\eta=0.550)$

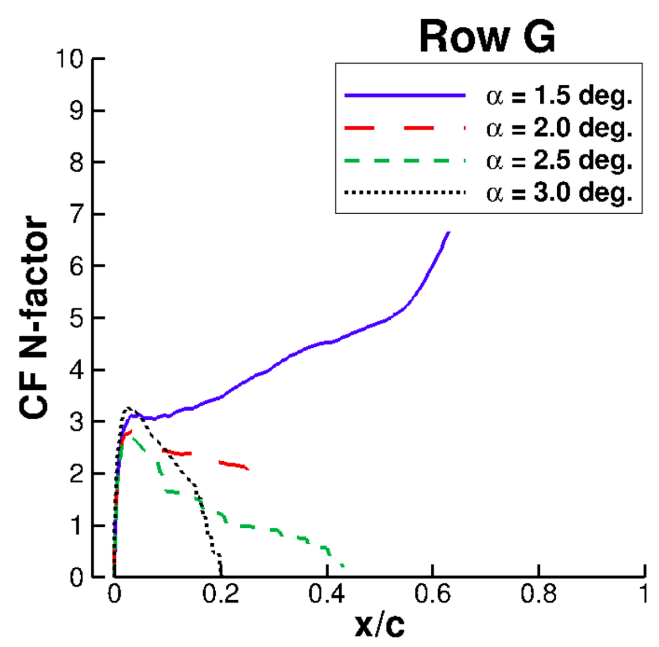

j) CF growth at $\operatorname{Row} G(\eta=0.730)$

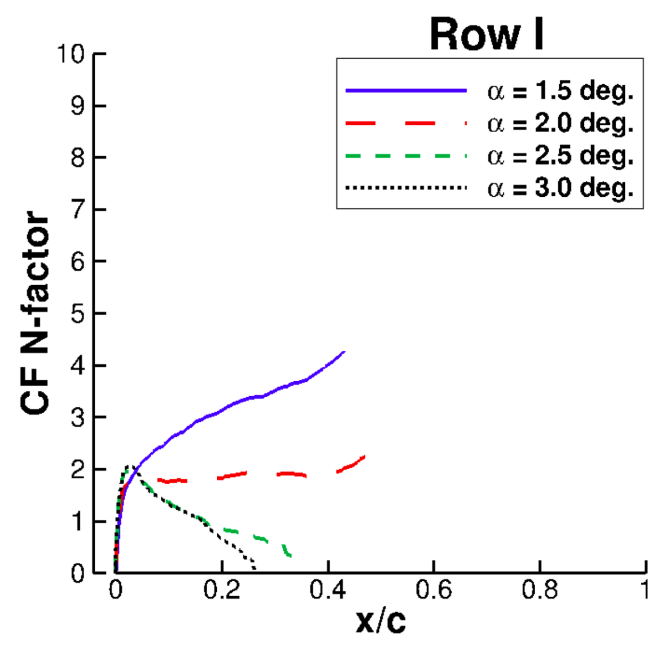

l) CF growth at Row $I(\eta=0.910)$

Figure 11 (continued). TS and CF N-factor growth versus $\mathrm{x} / \mathrm{c}$ at several spanwise stations for the angle-of-attack sweep from $M=0.86, T_{T}=40{ }^{\circ} \mathrm{F}$, and $\operatorname{Re}_{\mathrm{MAC}}=15$ million. 


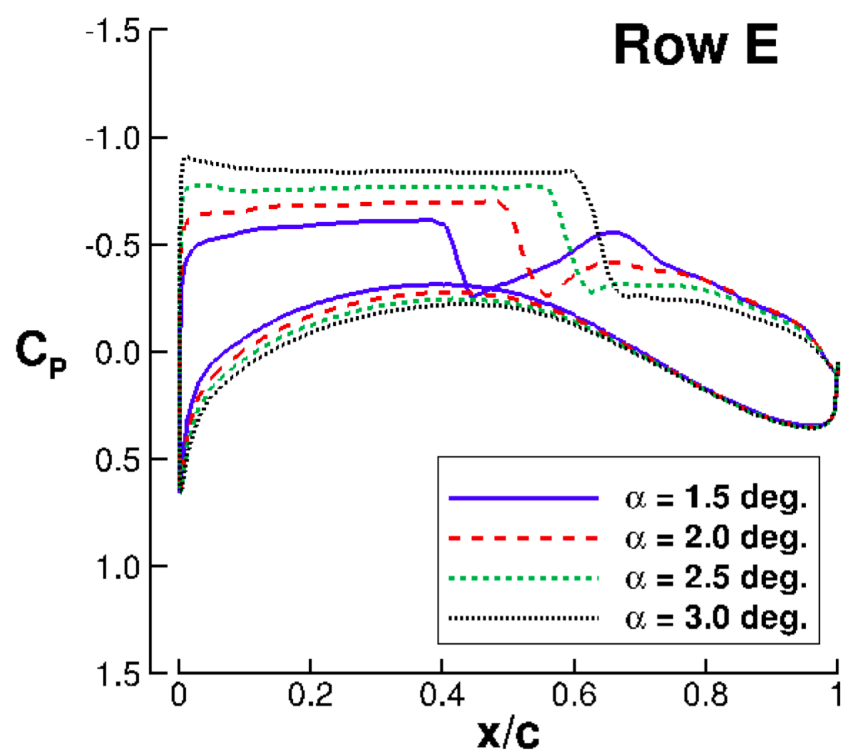

Figure 12. Computational pressure distributions at $\operatorname{Row} E(\eta=0.550)$ for the angle-of-attack $\operatorname{sweep}$ from $M=0.86, T_{T}=$ $40{ }^{\circ} \mathrm{F}$, and $\operatorname{Re}_{\mathrm{MAC}}=15$ million. 


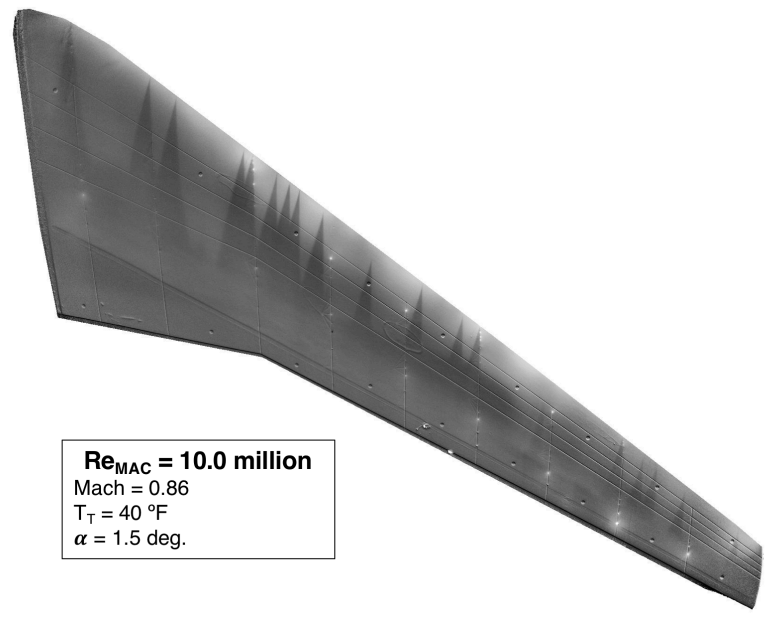

a) $\operatorname{Re}_{M A C}=10.0$ million

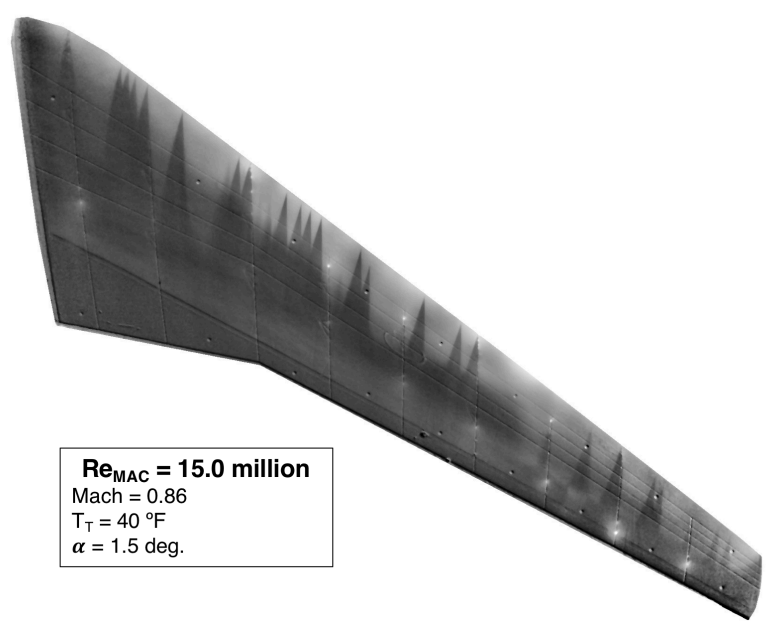

c) $\operatorname{Re}_{M A C}=15.0$ million

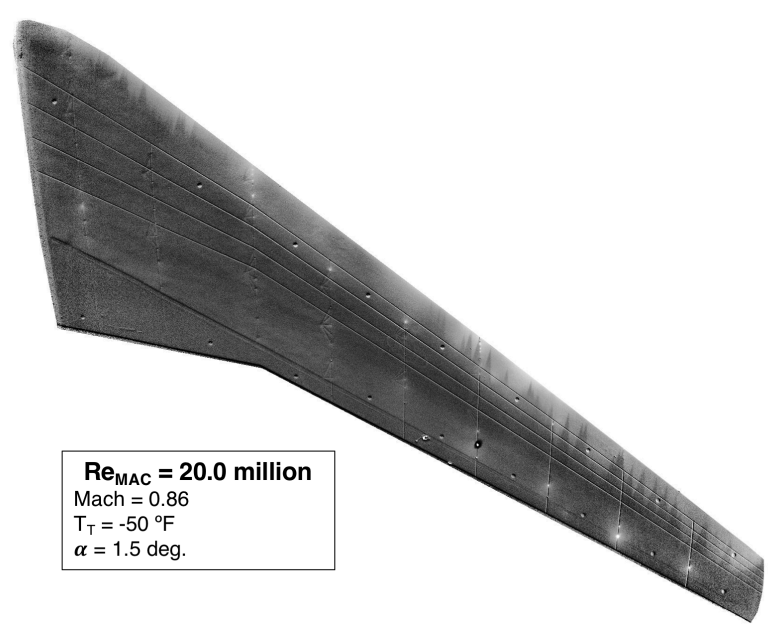

e) $\operatorname{Re}_{M A C}=\mathbf{2 0 . 0}$ million

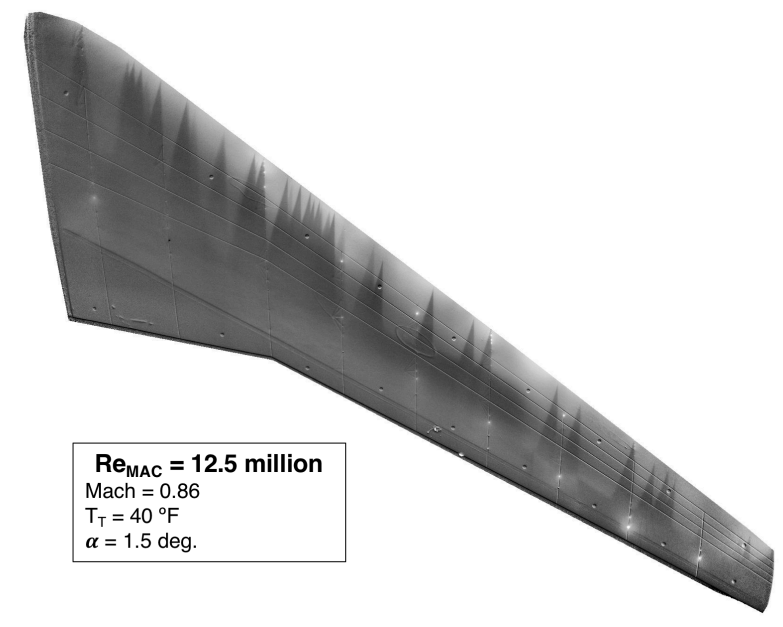

b) $\operatorname{Re}_{\text {MAC }}=12.5$ million

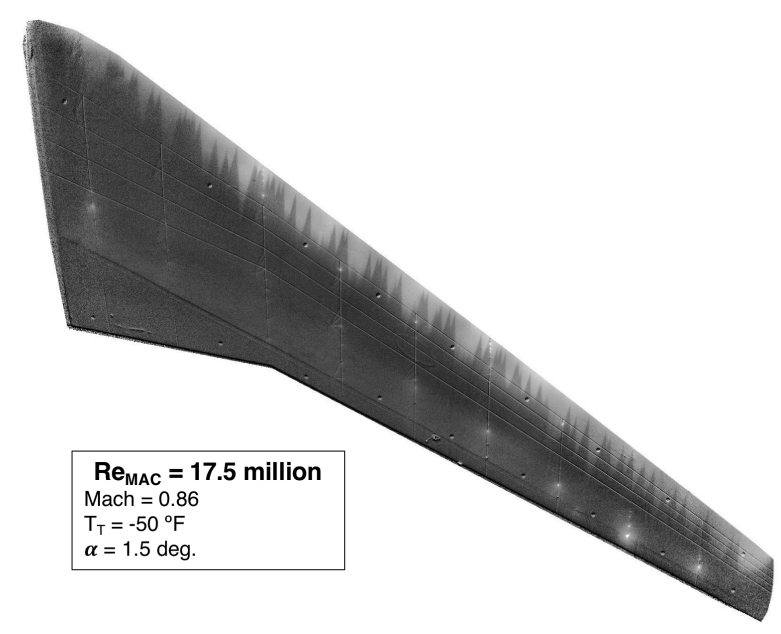

d) $\operatorname{Re}_{\mathrm{MAC}}=17.5$ million

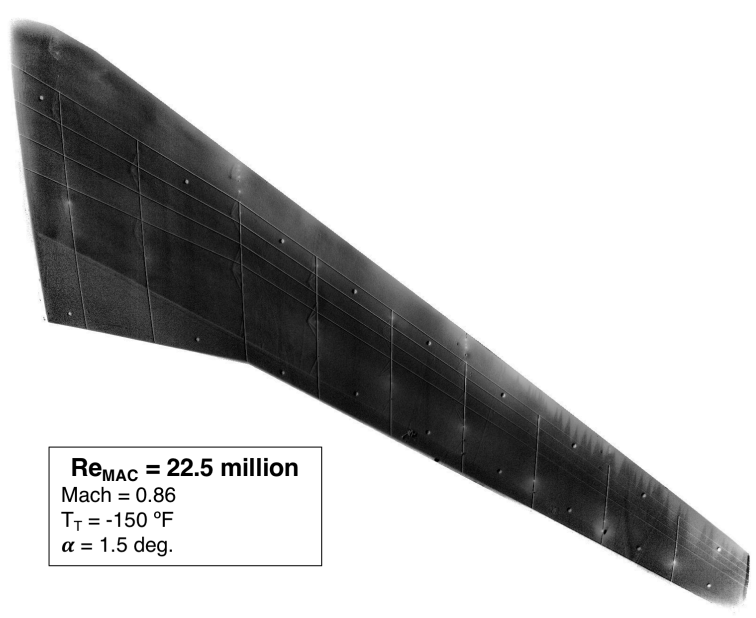

f) $\operatorname{Re}_{\mathrm{MAC}}=22.5$ million

Figure 13. TSP images from Reynolds number sweep at $M=0.86$ and $\alpha=1.5 \mathrm{deg}$. 


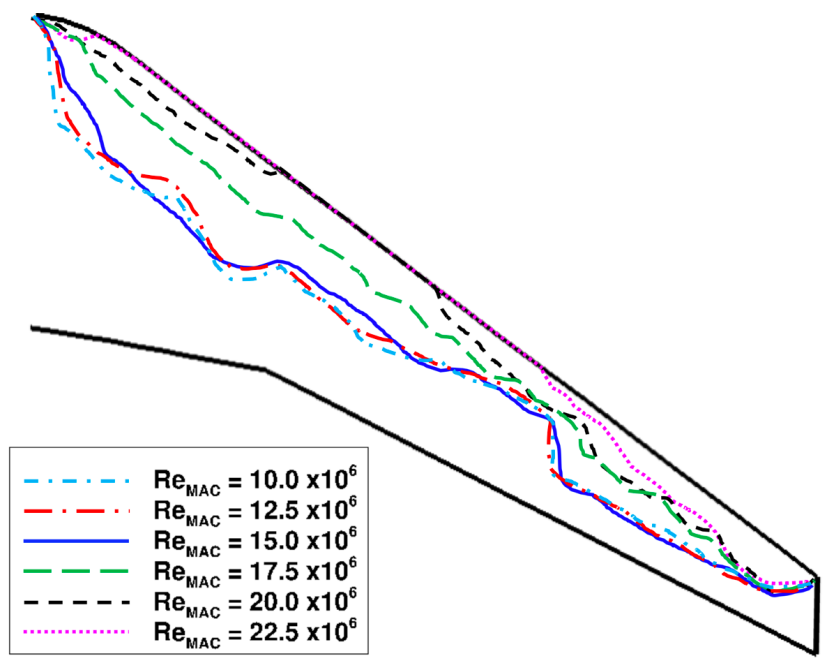

Figure 14. Experimental transition fronts from the Reynolds number sweep corresponding to the TSP images in Figure 13 at $M=0.86$ and $\alpha=1.5 \mathrm{deg}$. 


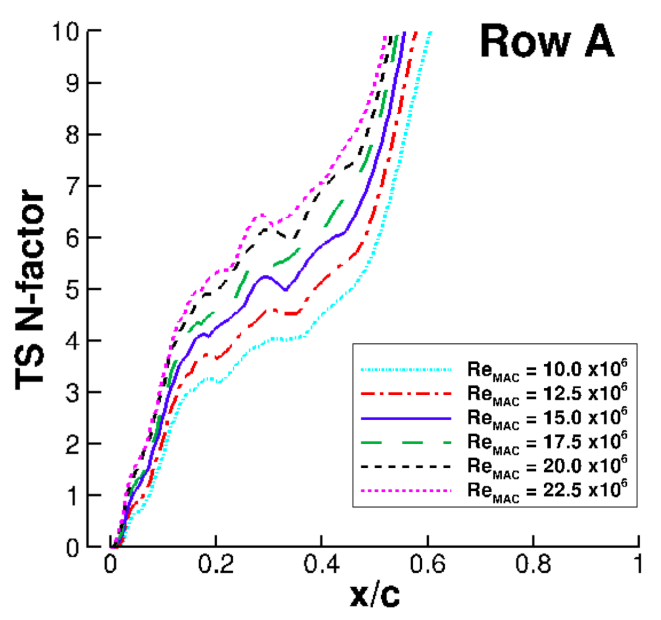

a) TS growth at $\operatorname{Row} A(\eta=0.163)$

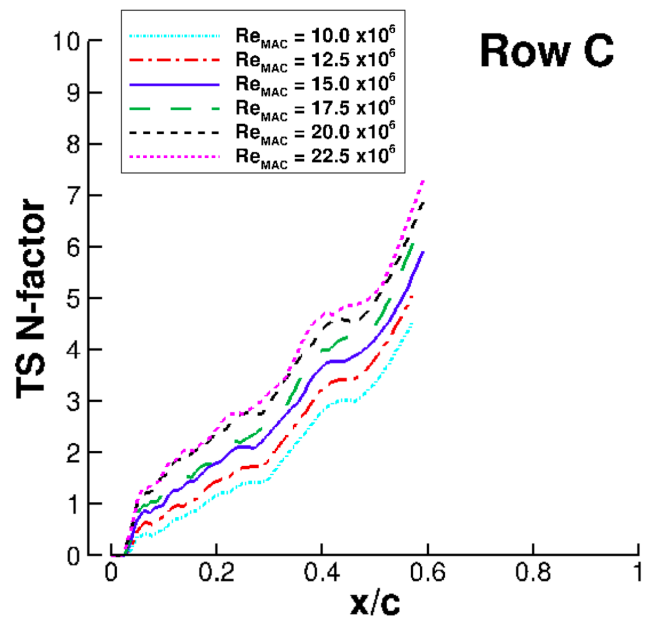

c) TS growth at Row $C(\eta=0.370)$

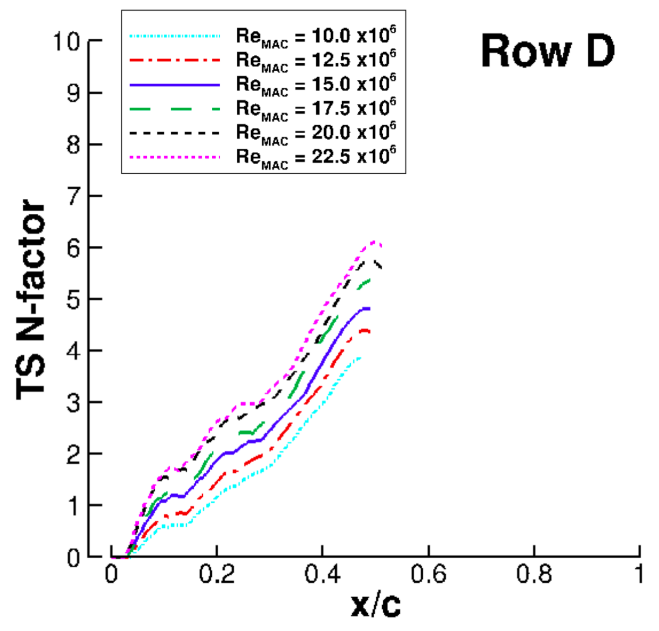

e) TS growth at Row $D(\eta=0.460)$

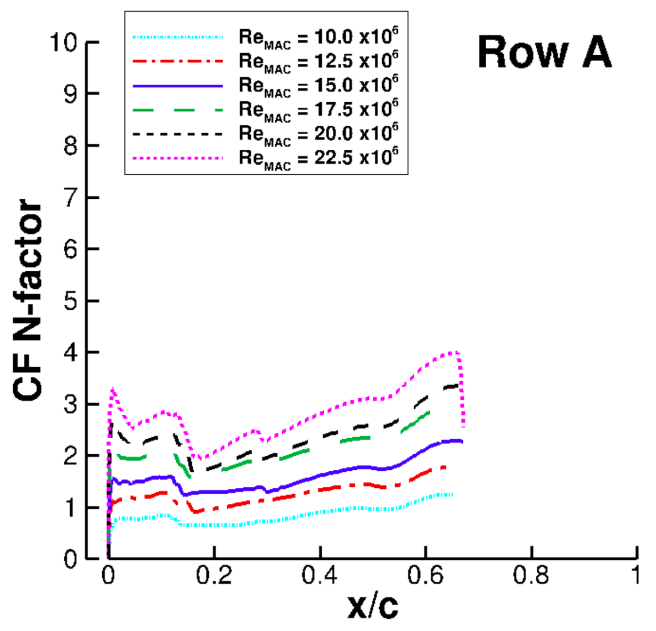

b) CF growth at $\operatorname{Row} A(\eta=0.163)$

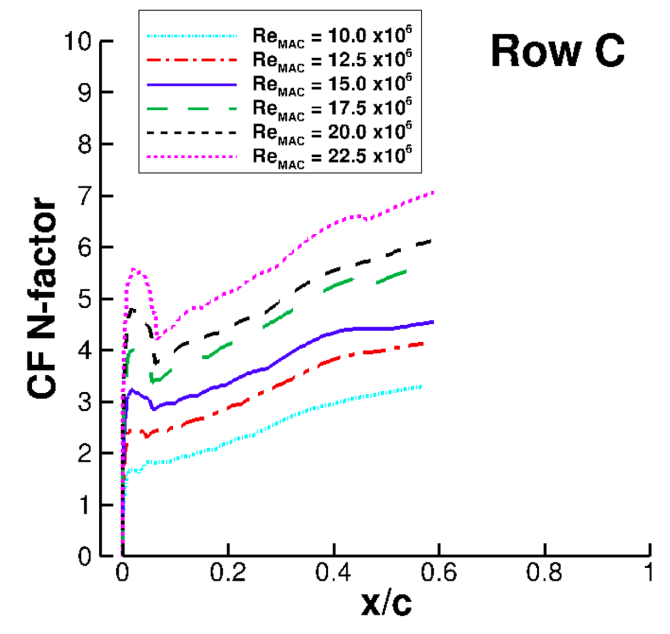

d) CF growth at Row C $(\eta=0.370)$

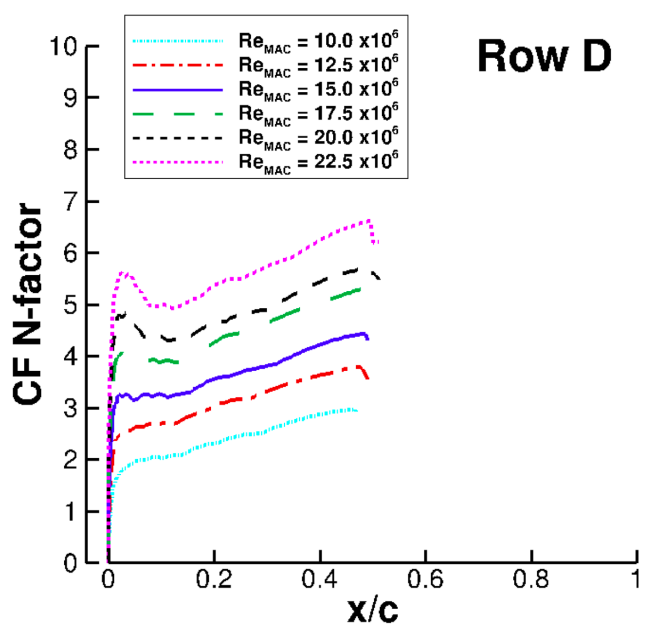

f) CF growth at $\operatorname{Row} D(\eta=0.460)$

Figure 15. TS and CF N-factor growth versus $\mathrm{x} / \mathrm{c}$ at several spanwise locations across the Reynolds number sweep from $M$ $=0.86$ and $\alpha=1.5 \mathrm{deg}$. 


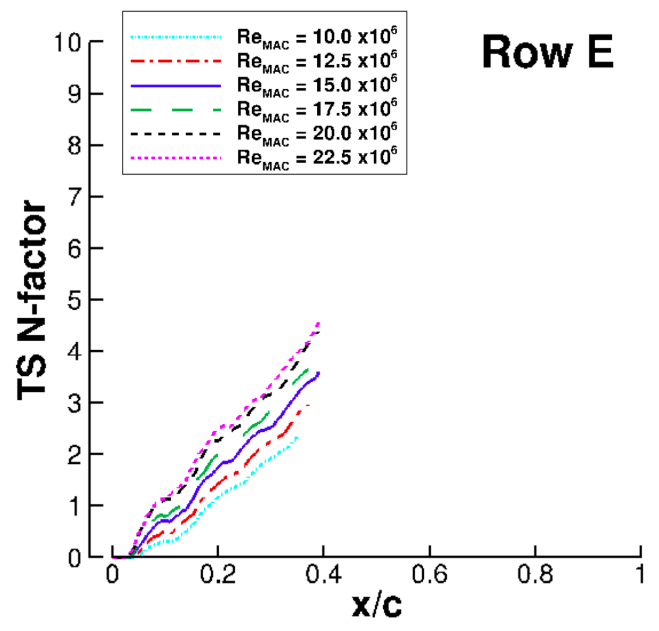

g) TS growth at $\operatorname{Row} E(\eta=0.550)$

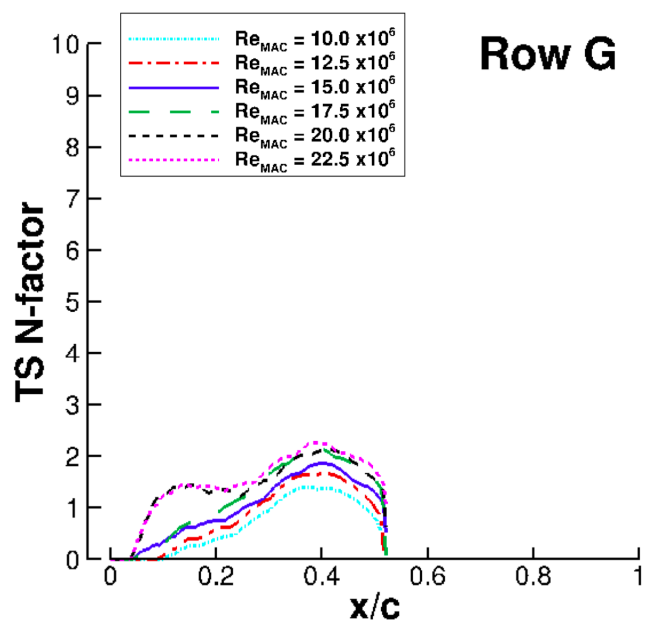

i) TS growth at $\operatorname{Row} G(\eta=0.730)$

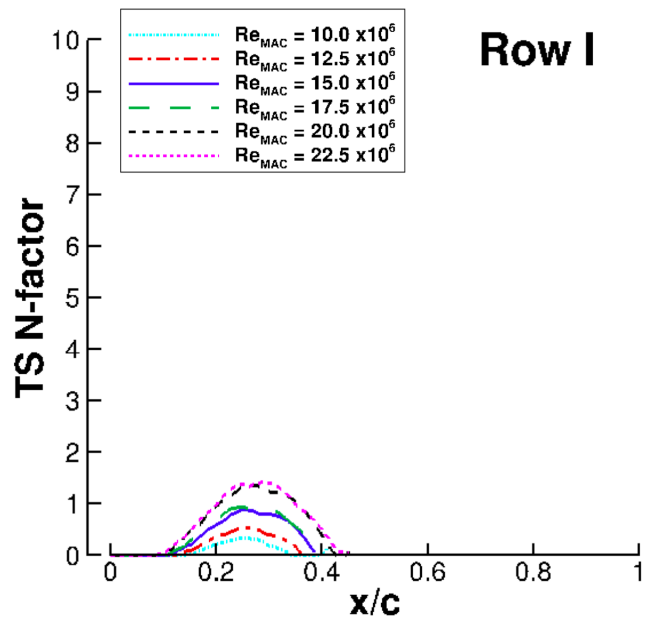

k) TS growth at $\operatorname{Row} I(\eta=\mathbf{0 . 9 1 0})$

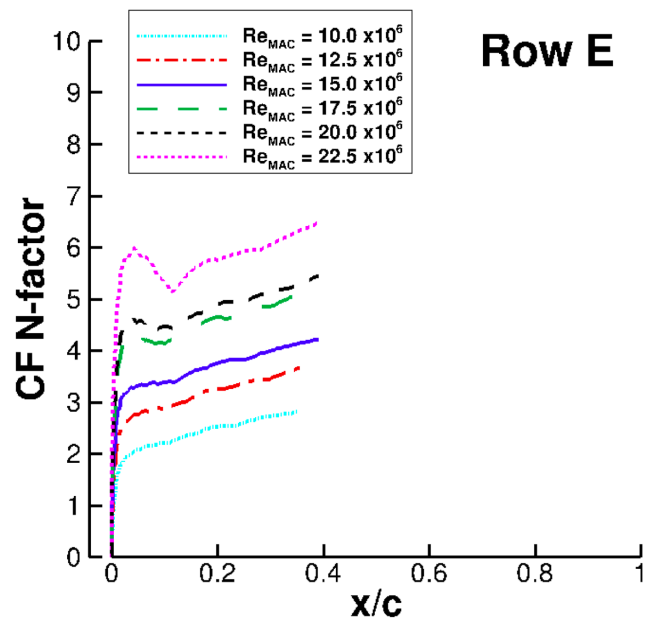

h) CF growth at $\operatorname{Row} E(\eta=0.550)$

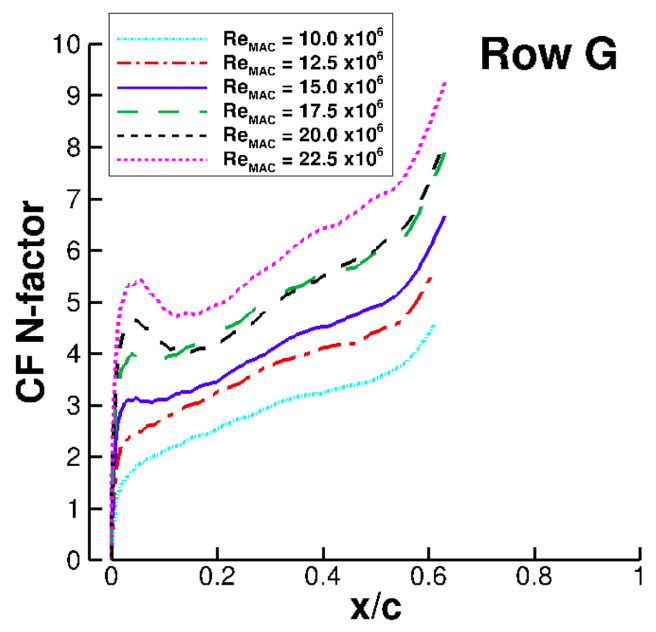

j) CF growth at $\operatorname{Row} G(\eta=0.730)$

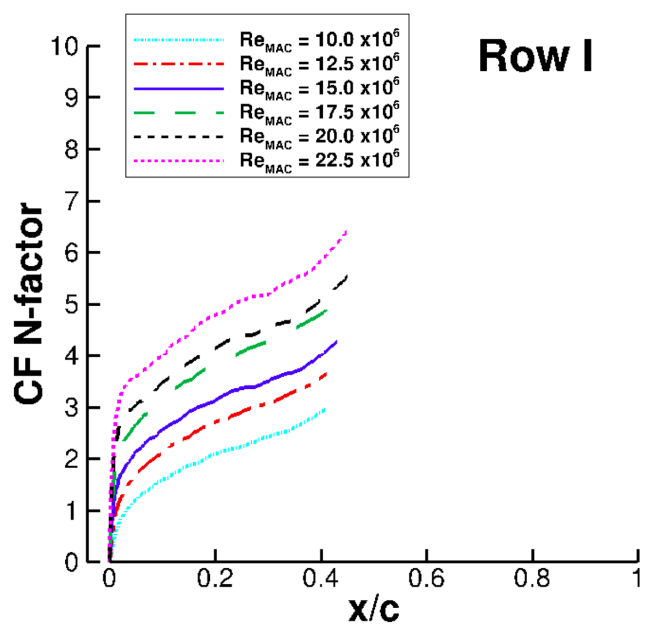

l) CF growth at $\operatorname{Row} I(\eta=0.910)$

Figure 15 (continued). TS and CF $\mathbf{N}$-factor growth versus $\mathbf{x} / \mathbf{c}$ at several spanwise locations across the Reynolds number sweep from $M=0.86$ and $\alpha=1.5 \mathrm{deg}$. 


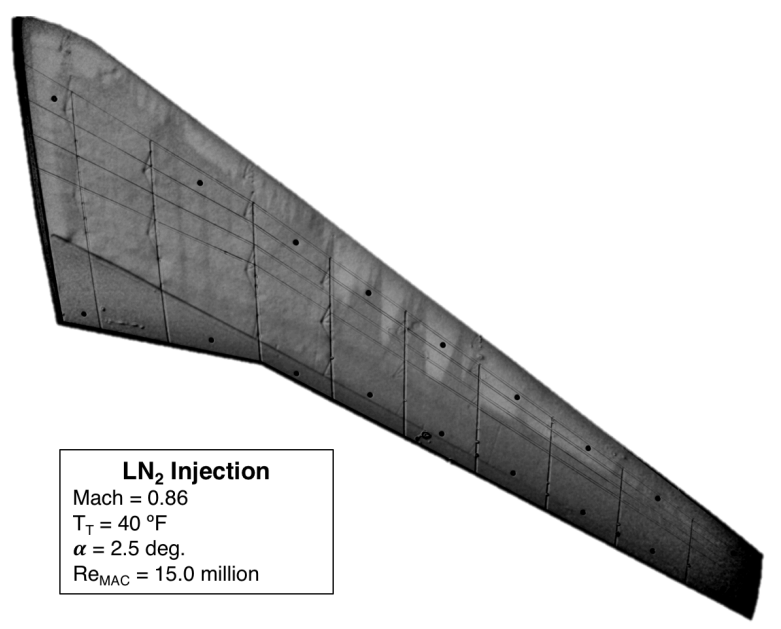

a) $\mathrm{LN}_{2}$ injection method

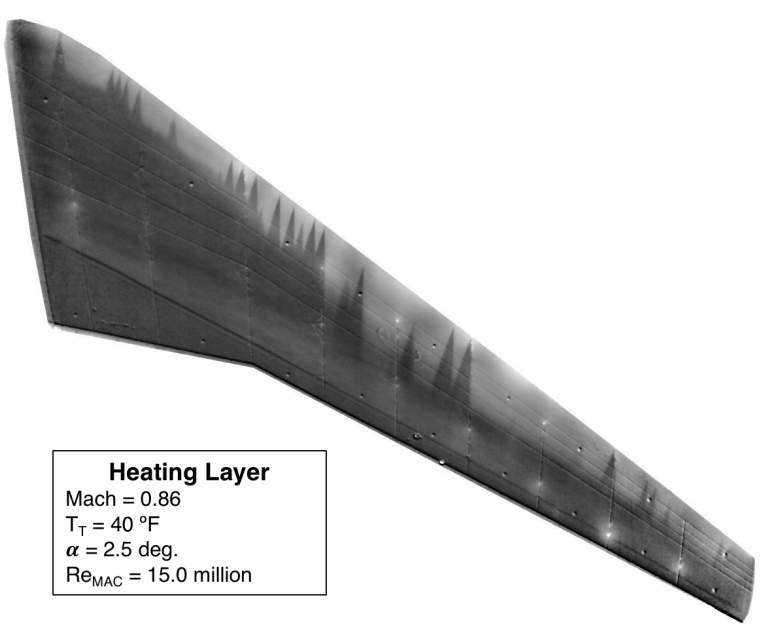

b) Heating layer method

Figure 16. TSP images from the two different temperature gradient mechanisms. Both images are from $M=0.86, \alpha=2.5$ deg., $T_{T}=40^{\circ} \mathrm{F}$, and $\operatorname{Re}_{\mathrm{MAC}}=\mathbf{1 5 . 0}$ million.

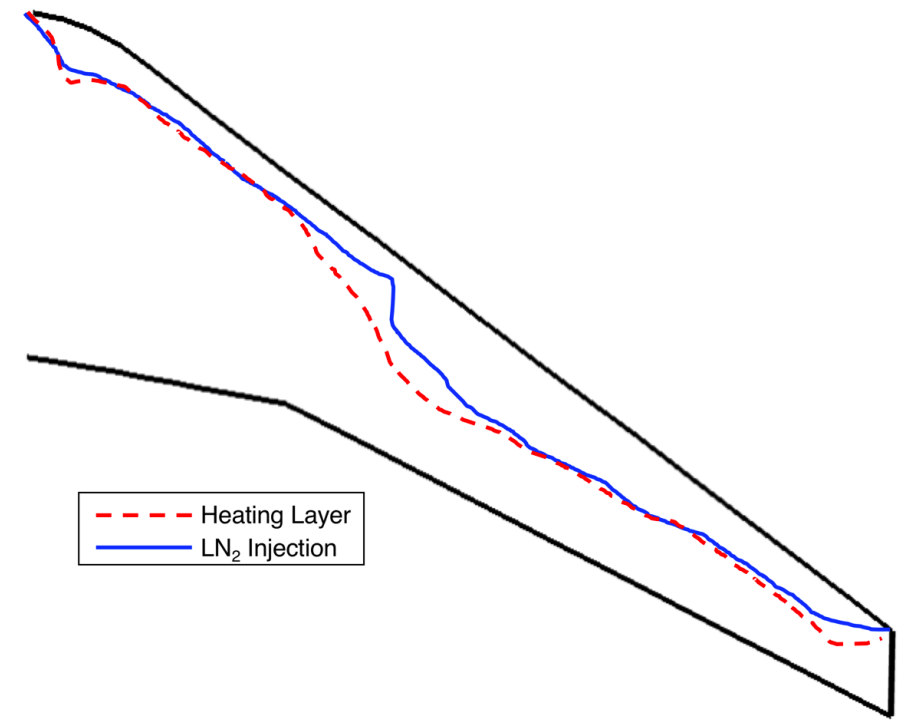

Figure 17. Experimental transition fronts from the two temperature gradient mechanisms corresponding to the TSP images in Figure 16 at $M=0.86, \alpha=2.5 \mathrm{deg}$., $T_{T}=40^{\circ} \mathrm{F}$, and $\operatorname{Re}_{\mathrm{MAC}}=15.0$ million. 


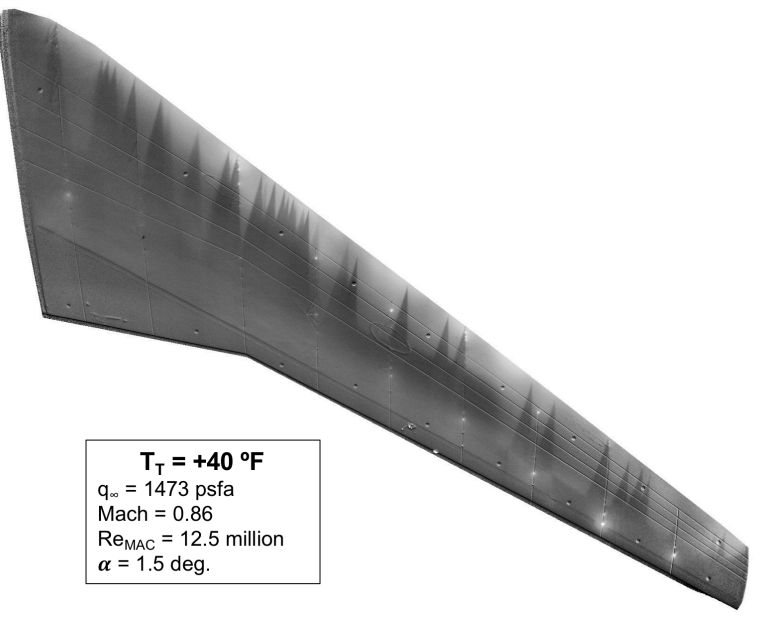

a) $T_{T}=40^{\circ} \mathrm{F}$ and $q_{\infty}=1473 \mathrm{psfa}$

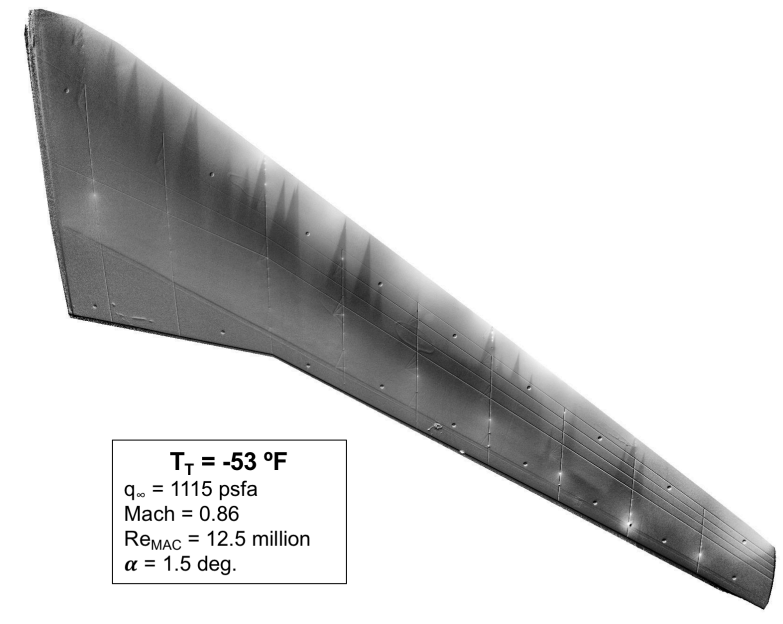

b) $T_{T}=-53{ }^{\circ} \mathrm{F}$ and $q_{\infty}=1115 \mathrm{psfa}$

Figure 18. TSP images from the two different tunnel temperature/pressure combinations. Both images are from $M=0.86$, $\alpha=1.5$ deg., and $\operatorname{Re}_{\mathrm{MAC}}=12.5$ million.

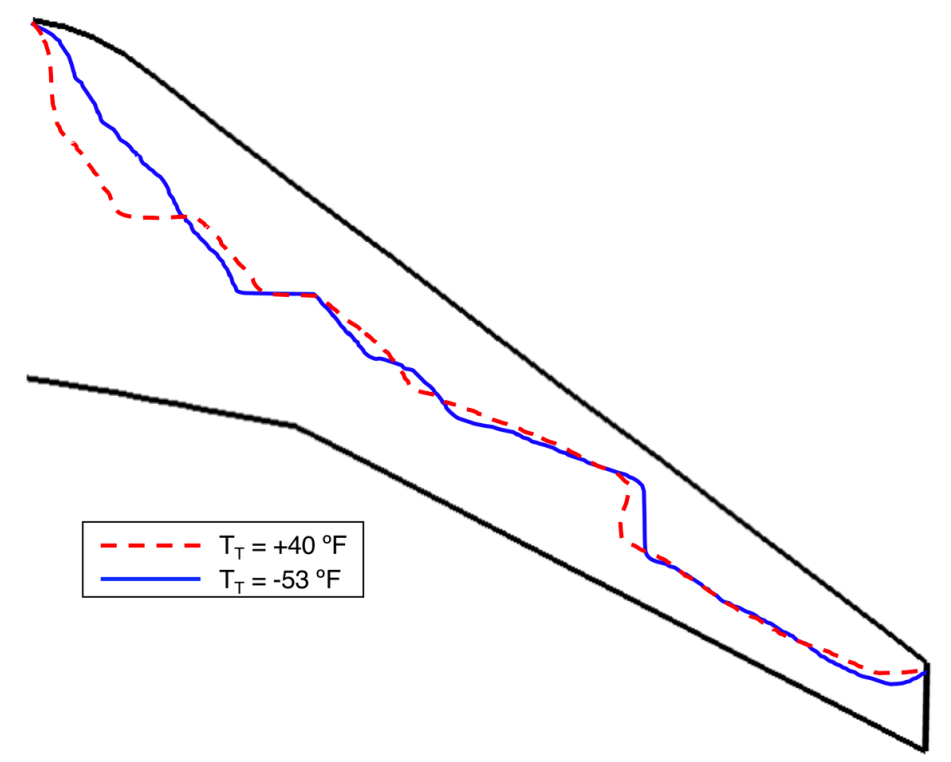

Figure 19. Experimental transition fronts from the two temperature/pressure combinations corresponding to the TSP images in Figure 18 at $M=0.86, \alpha=1.5$ deg., and $\operatorname{Re}_{M A C}=12.5$ million. 


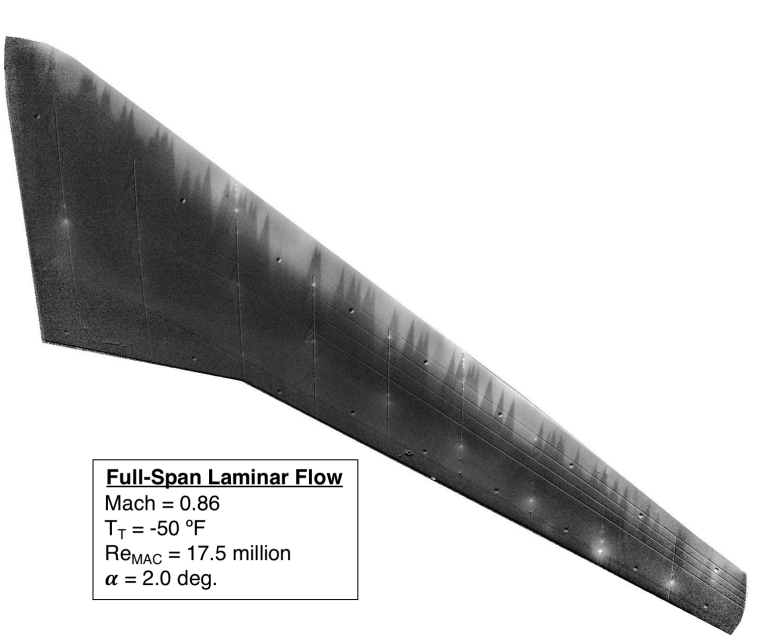

a) Full-span laminar flow

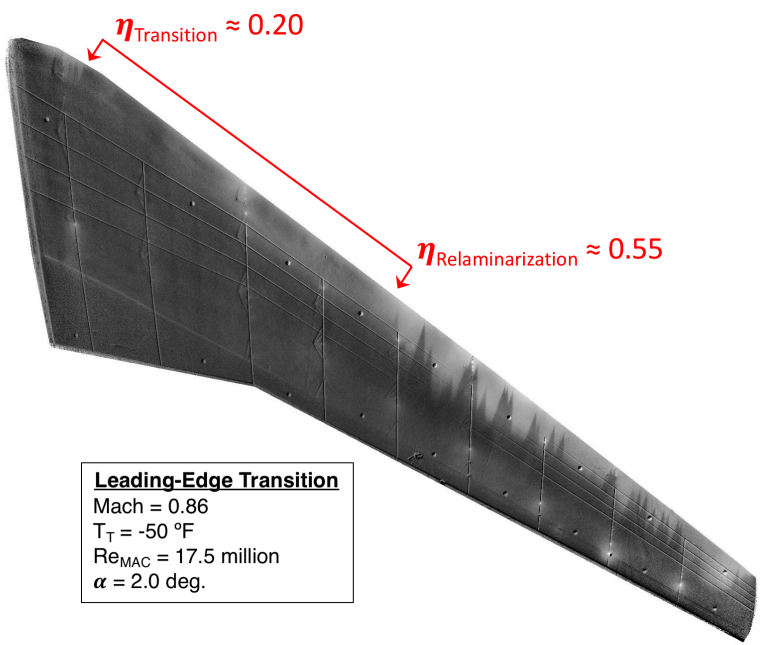

b) Leading-edge transition

Figure 20. TSP images from repeat points of the same nominal tunnel conditions of $M=0.86, T_{T}=-53{ }^{\circ} \mathbf{F}, \alpha=2.0$ deg., and $R_{M A C}=17.5$ million. One image shows full-span laminar flow (a) and the other has leading-edge transition (b). The semispan locations of leading-edge transition and leading-edge relaminarization are also shown.

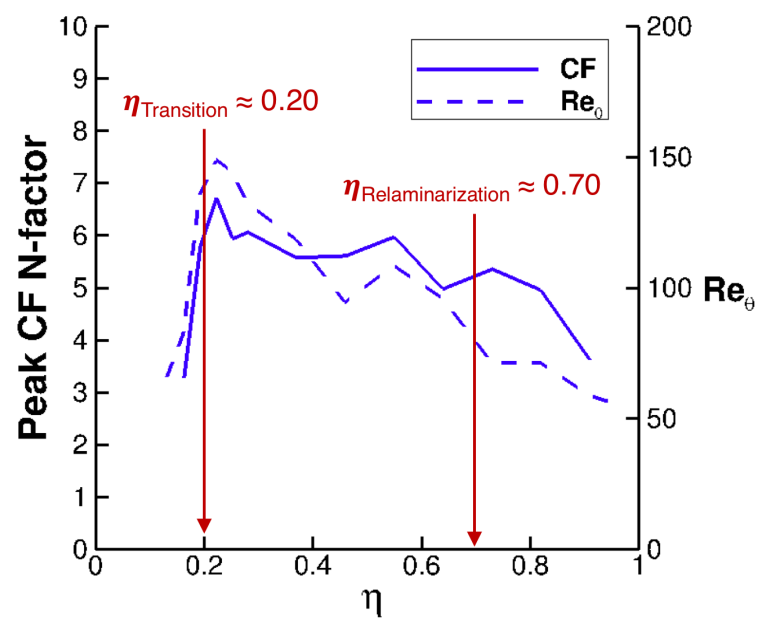

Figure 21. Computational attachment line $\operatorname{Re}_{\theta}$ values out the semispan for the condition shown in Figure $20\left(M=0.86, T_{T}\right.$ $=-53{ }^{\circ} \mathrm{F}, \alpha=2.0 \mathrm{deg}$, and $\left.\operatorname{Re}_{\mathrm{MAC}}=17.5 \mathrm{million}\right)$. The semispan locations of leading-edge transition and leading-edge relaminarization are noted on the plot, along with the $\operatorname{Re}_{\theta}$ value at the relaminarization location. 


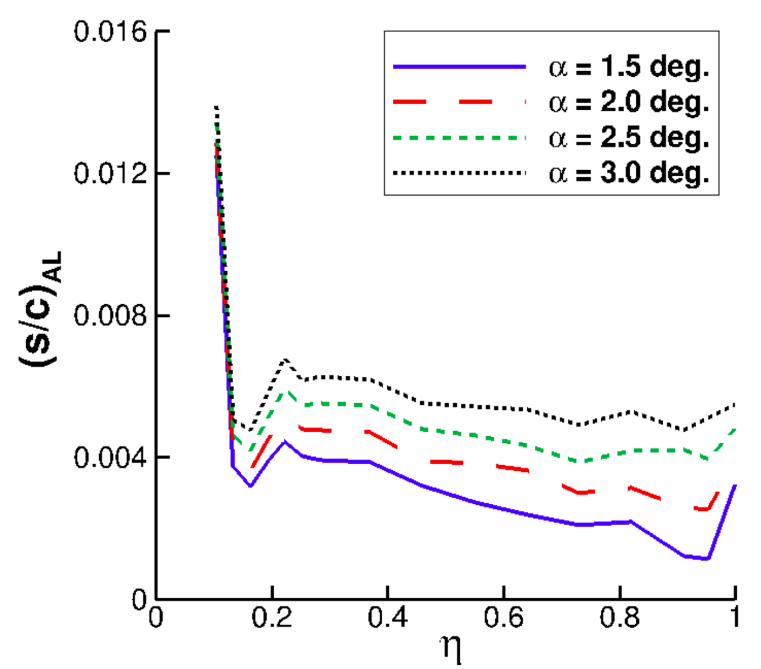

a) Angle-of-attack sweep at $\operatorname{Re}_{\mathrm{MAC}}=15$ million

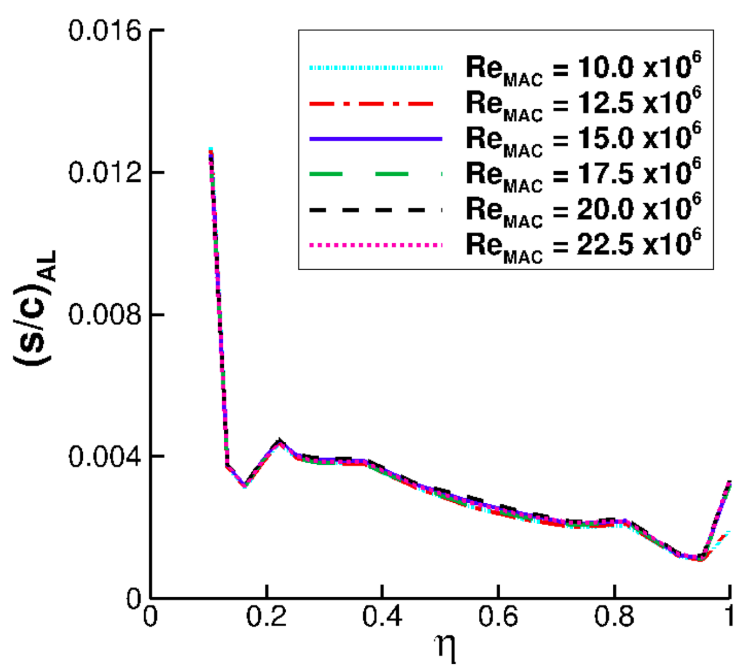

b) Reynolds number sweep at $\alpha=1.5 \mathrm{deg}$.

Figure 22. Arc length location of the attachment line across the semispan for both the angle-of-attack and Reynolds number sweeps. All data are from computational results from $\mathrm{M}=\mathbf{0 . 8 6}$.

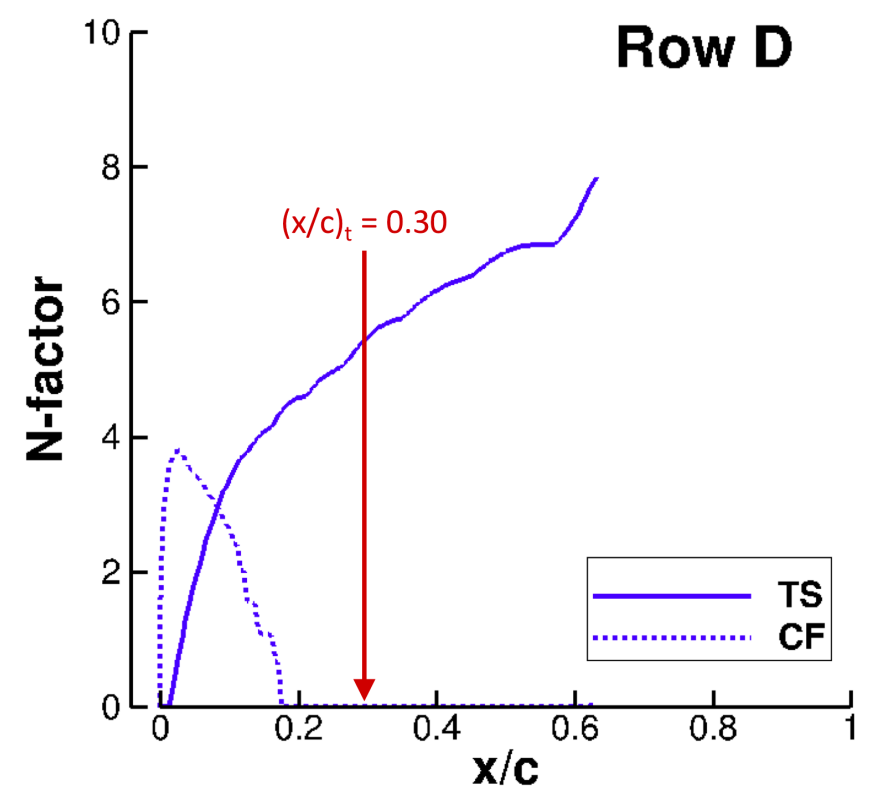

Figure 23. N-factor growth for TS (solid) and CF (dashed) with experimental transition location noted, showing TS is likely the dominant transition mechanism at $\operatorname{Row} D(\eta=0.460)$. Computational results correspond to nominal conditions of $M=0.86, T_{T}=40^{\circ} \mathrm{F}, \alpha=3.0 \mathrm{deg}$., and $\operatorname{Re}_{\mathrm{MAC}}=15.0 \mathrm{million}$. 


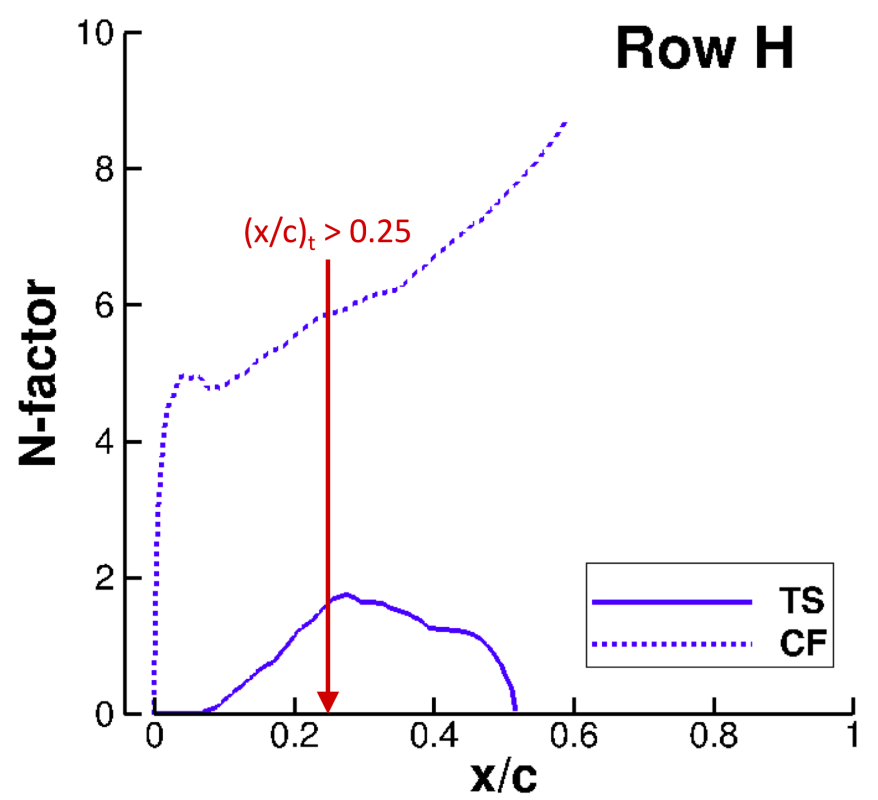

Figure 24. N-factor growth for TS (solid) and CF (dashed) with experimental wedge coalescence location noted, showing CF is likely the dominant transition mechanism at Row $H(\eta=0.820)$. Computational results correspond to nominal conditions of $M=0.86, T_{T}=-153{ }^{\circ} \mathrm{F}, \alpha=1.5 \mathrm{deg}$., and $\operatorname{Re}_{\mathrm{MAC}}=22.5$ million.

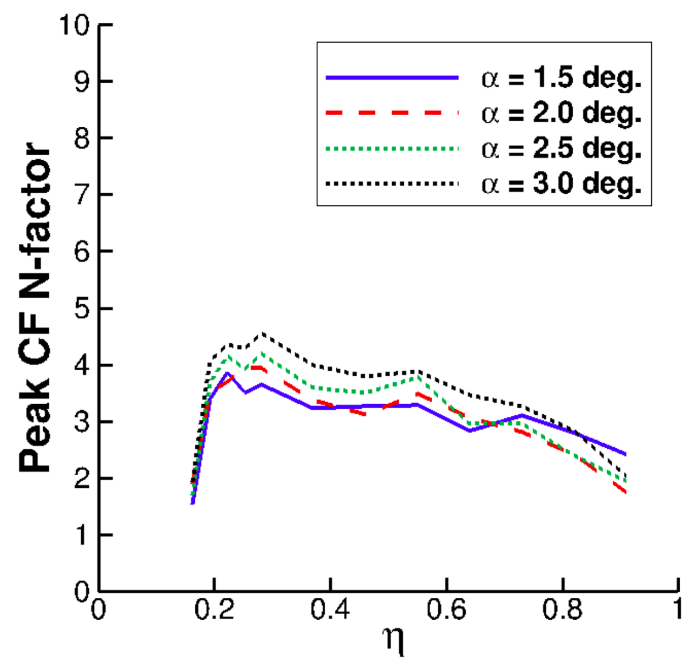

a) Angle-of-attack sweep at $\operatorname{Re}_{\mathrm{MAC}}=15$ million

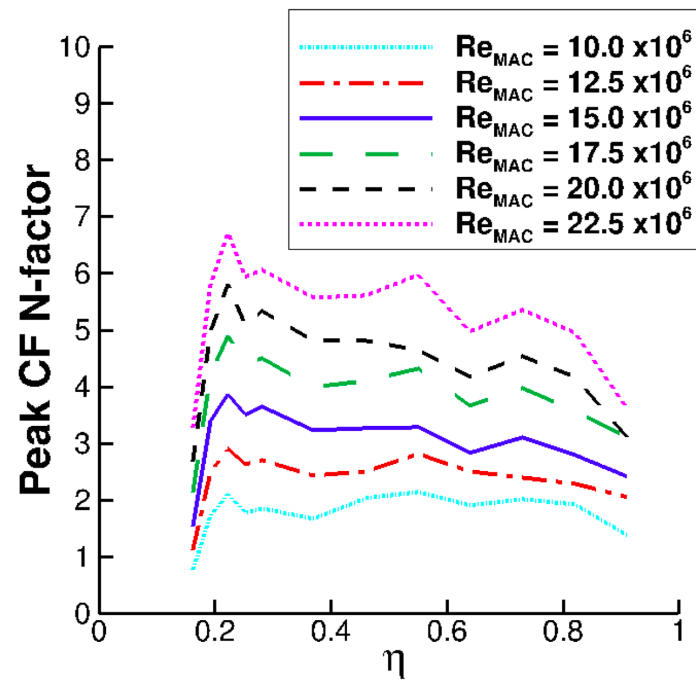

b) Reynolds number sweep at $\alpha=1.5 \mathrm{deg}$.

Figure 25. Peak CF N-factor across the semispan for both the angle-of-attack and Reynolds number sweeps. All data are from computational results from nominal $M=0.86$. 


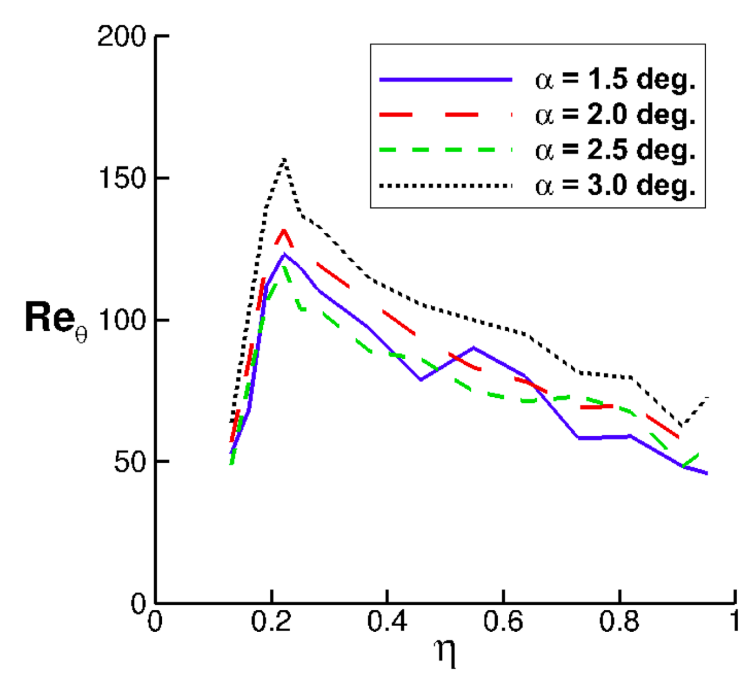

a) Angle-of-attack sweep at $\operatorname{Re}_{\mathrm{MAC}}=15$ million

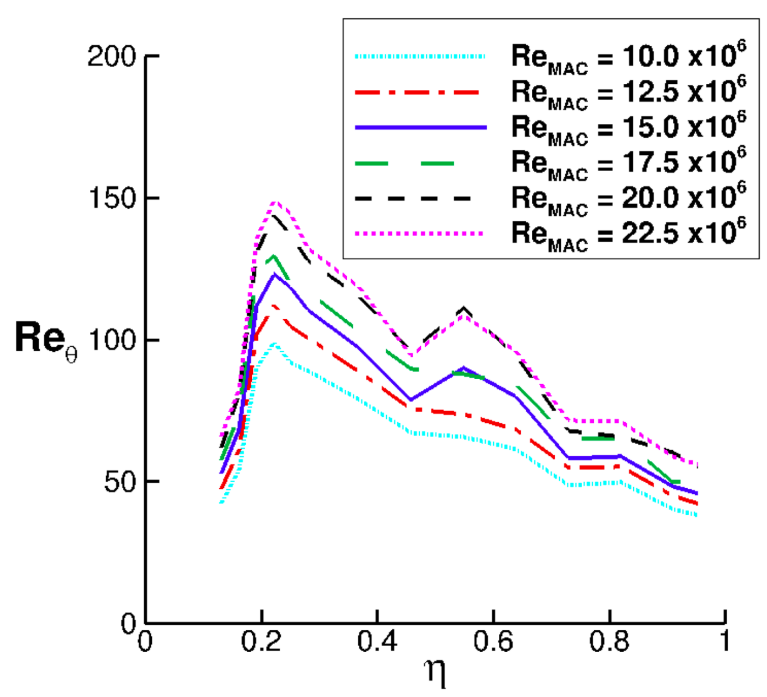

b) Reynolds number sweep at $\alpha=1.5 \mathrm{deg}$.

Figure 26. Computational attachment line $\operatorname{Re}_{\theta}$ values across the semispan for both the angle-of-attack and Reynolds number sweeps from nominal $M=0.86$.

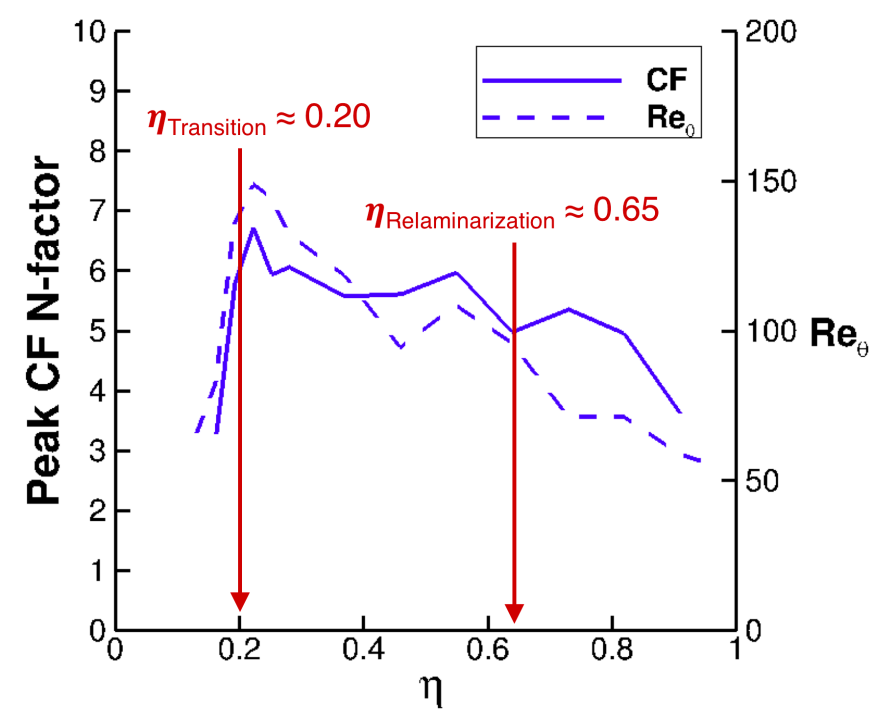

Figure 27. Computational peak $\mathrm{CF} N$-factors and attachment line $\mathrm{Re}_{\theta}$ values across the semispan with the semispan location of leading-edge transition and leading-edge relaminarization noted. Results correspond to nominal conditions of $M=0.86, T_{T}=-153{ }^{\circ} \mathrm{F}, \alpha=1.5$ deg., and $\operatorname{Re}_{\mathrm{MAC}}=22.5$ million. 


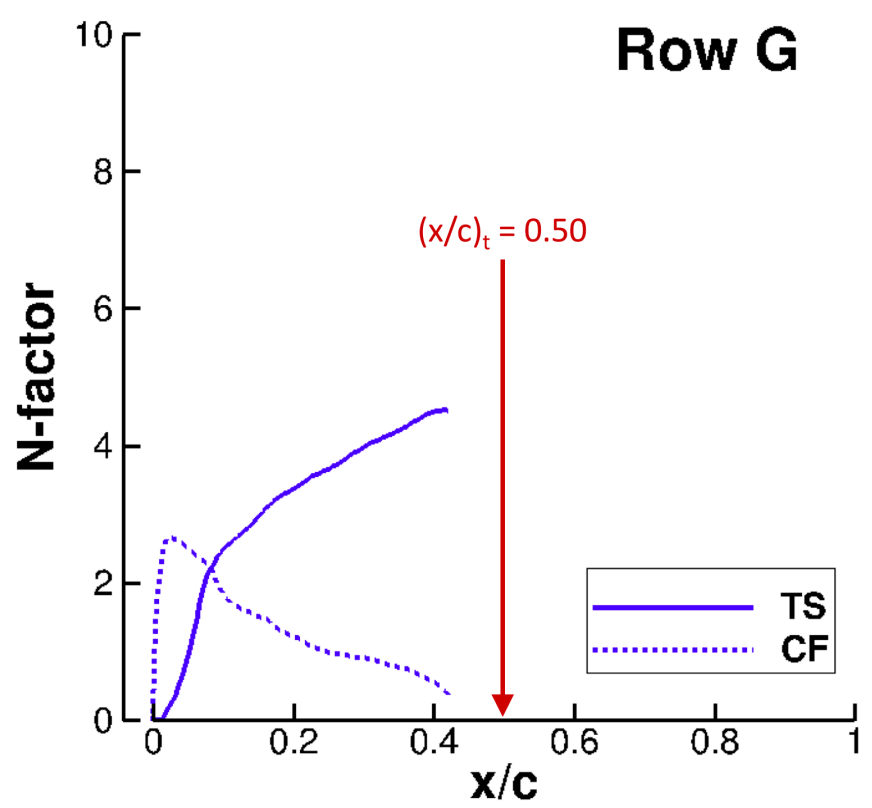

Figure 28. $N$-factor growth for TS (solid) and CF (dashed) versus $x / c$ at Row $G(\eta=0.730)$ from nominal conditions of $M$ $=0.86, T_{T}=40^{\circ} \mathrm{F}, \alpha=2.5 \mathrm{deg}$., and $\operatorname{Re}_{\mathrm{MAC}}=\mathbf{1 5 . 0}$ million. The plot shows the experimental transition location is aft of the extent of $\mathbf{N}$-factor computations.

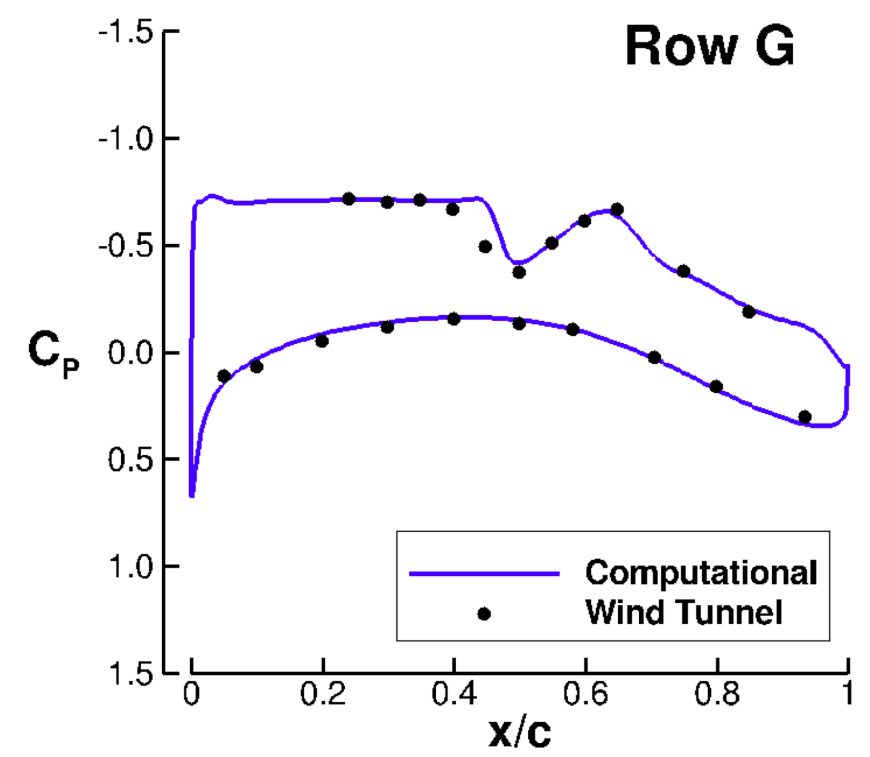

Figure 29. Pressure distributions from computational results and wind tunnel pressures at $\operatorname{Row} G(\eta=0.730)$ from nominal conditions of $M=0.86, T_{T}=40^{\circ} \mathrm{F}, \alpha=2.5 \mathrm{deg}$., and $\operatorname{Re}_{M A C}=15.0$ million. The plot shows a pressure gradient (shock) seen forward of the experimental transition location of $(x / c)_{t}=0.50$, explaining why the $N$-factor growth in Figure 28 terminates early. 


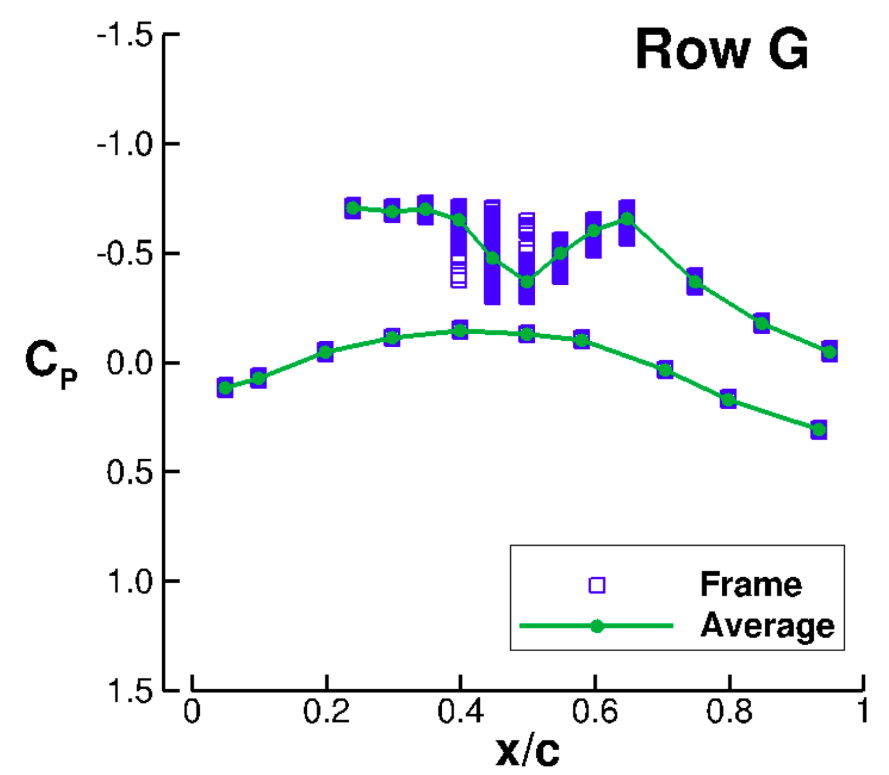

Figure 30. Pressure distribution of experimental pressure data at $\operatorname{Row} G(\eta=0.730)$ from nominal conditions of $M=0.86$, $T_{T}=40^{\circ} \mathrm{F}, \alpha=2.5 \mathrm{deg}$., and $\operatorname{Re}_{\mathrm{MAC}}=15.0$ million. Every pressure data frame (blue squares) required during data acquisition is shown with the average data (green) overlaid.

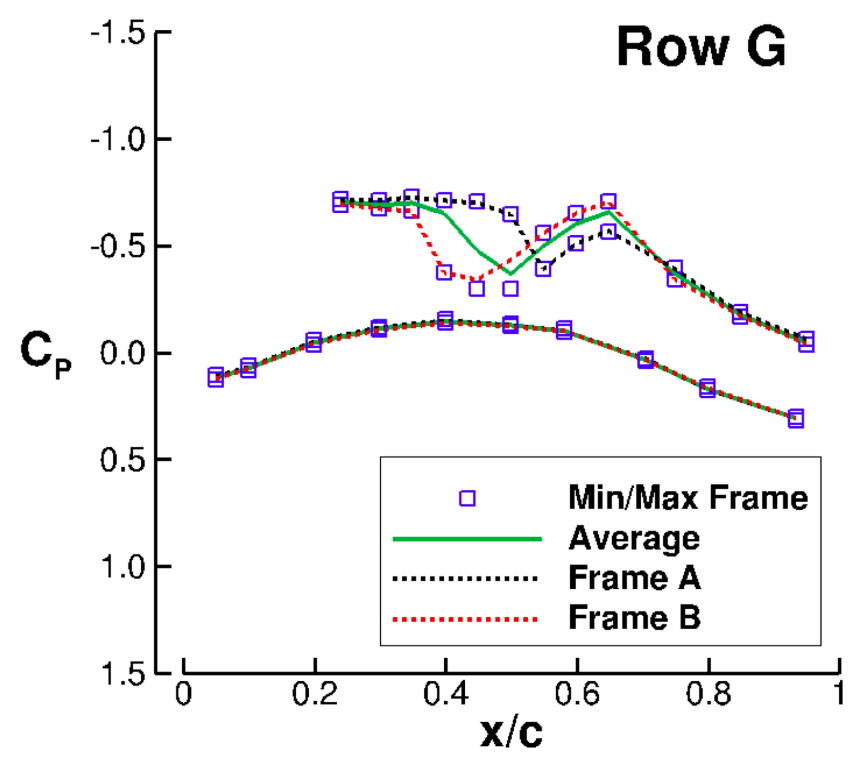

Figure 31. Pressure distribution of experimental pressure data at $\operatorname{Row} G(\eta=0.730)$ from nominal conditions of $M=0.86$, $T_{T}=40^{\circ} \mathrm{F}, \alpha=2.5 \mathrm{deg}$., and $\operatorname{Re}_{\mathrm{MAC}}=\mathbf{1 5 . 0}$ million. The average experimental pressures (green) and the minimum and maximum values at each pressure port from all frames over the data acquisition (blue squares) are shown. Two individual frames (Frame $A$ and Frame B) are also shown that correspond to the most aft shock (Frame A) and a collapsed rooftop pressure distribution (Frame B), showing that the large variation in pressure port readings between $0.35<\mathrm{x} / \mathrm{c}<0.55$ are likely related to the rooftop pressures collapsing during the data acquisition. 


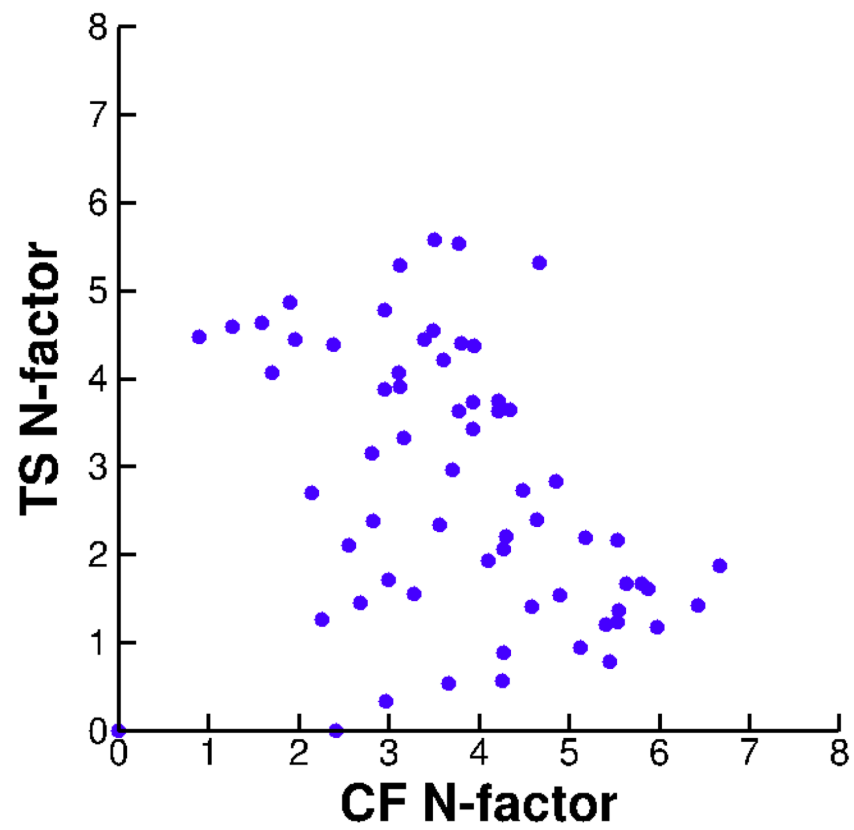

Figure 32. N-factor values of TS and CF at the experimental transition location for all data analyzed in the angle-ofattack and Reynolds number sweeps.

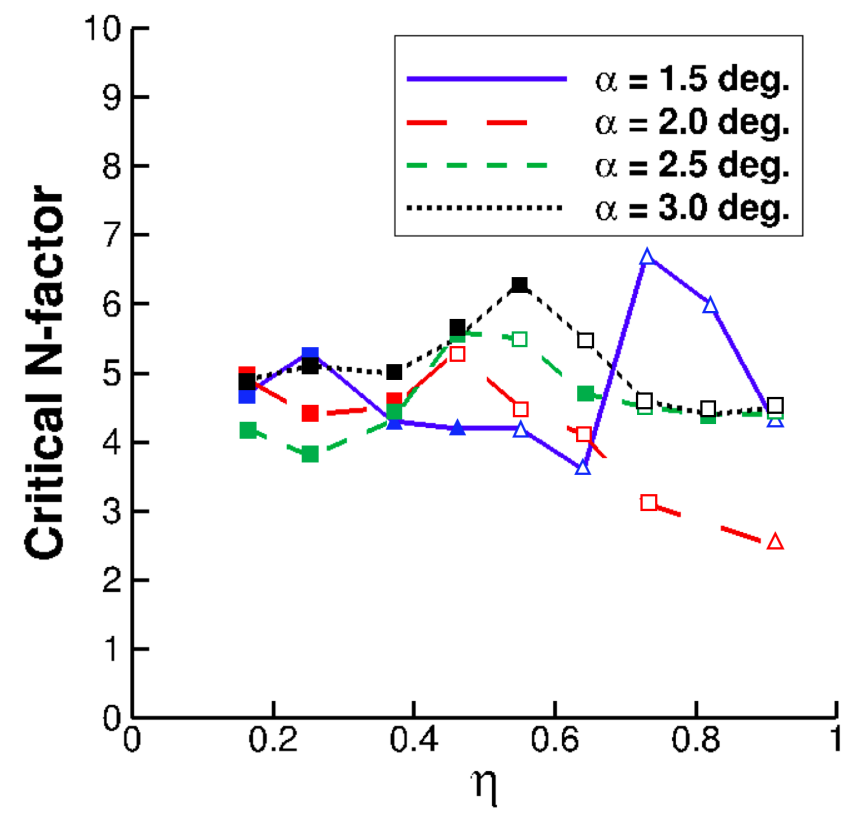

Figure 33. Critical $\mathrm{N}$-factor values across the semispan for the angle-of-attack sweep at nominal conditions of $\mathrm{M}=\mathbf{0 . 8 6}$, $T_{T}=40{ }^{\circ} \mathbf{F}$, and $\operatorname{Re}_{M A C}=15.0$ million. Square symbols are when TS was the dominant mechanism and triangle symbols are when $\mathrm{CF}$ was the dominant mechanism. Filled symbols indicate there was $\mathrm{N}$-factor calculations at the experimental transition location, and open symbols indicate the $\mathrm{N}$-factor calculations terminated forward of the experimental transition location. Open symbols are likely artificially low due to the early termination of $\mathrm{N}$-factor calculations. 


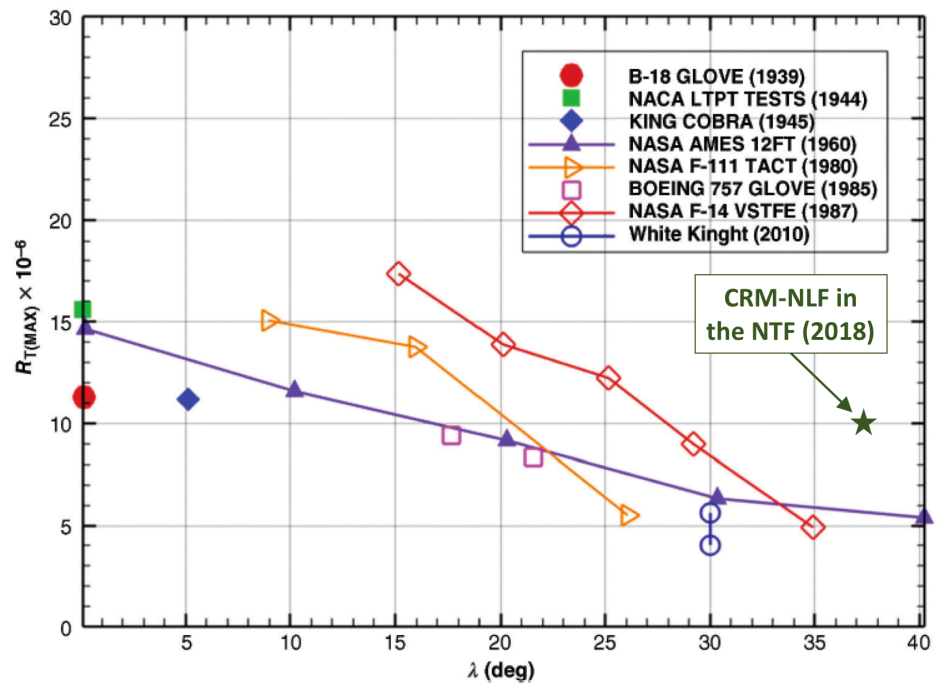

Figure 34. Graph by Malik et al. showing the maximum transition Reynolds number $\left(R_{T(\max )}\right)$ as a function of wing sweep ( $\lambda$ ) for a variety of NLF experiments [27]. Overlaid on the plot is the maximum finding of the CRM-NLF in the NTF showing the high transition Reynolds numbers achieved during the test relative to historic NLF experiments.

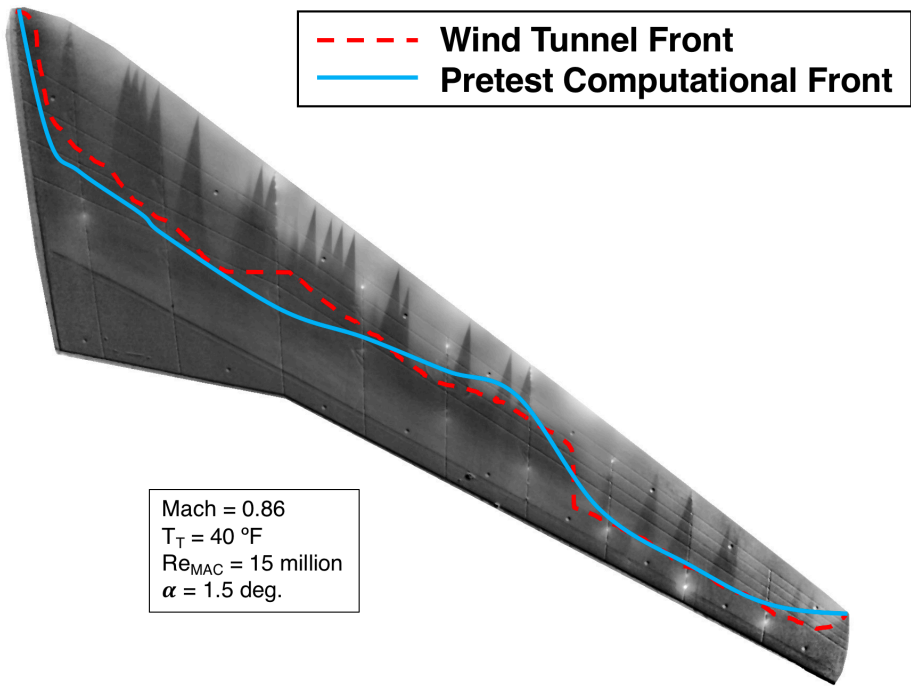

Figure 35. TSP image with the approximated transition front shown (red dashed line) from nominal test conditions of $M$ $=0.86, T_{T}=40{ }^{\circ} \mathrm{F}, \alpha=1.5 \mathrm{deg}$., and $\operatorname{Re}_{\mathrm{MAC}}=15.0$ million. The pretest computational transition predictions at corresponding conditions for a critical $\mathbf{N}$-factor of 6 is overlaid on the image (blue solid line). 